\title{
Simple Tests for Models of Dependence Between Multiple Financial Time Series, with Applications to U.S. Equity Returns and Exchange Rates*
}

\author{
Xiaohong Chen \\ New York University
}

\author{
Yanqin Fan \\ Vanderbilt University
}

\author{
Andrew Patton \\ London School of Economics
}

First version: May 2003, This version: January 2004

\begin{abstract}
Evidence that asset returns are more highly correlated during volatile markets and during market downturns (see Longin and Solnik, 2001, and Ang and Chen, 2002) has lead some researchers to propose alternative models of dependence. In this paper we develop two simple goodness-of-fit tests for such models. We use these tests to determine whether the multivariate Normal or the Student's $t$ copula models are compatible with U.S. equity return and exchange rate data. Both tests are robust to specifications of marginal distributions, and are based on the multivariate probability integral transform and kernel density estimation. The first test is consistent but requires the estimation of a multivariate density function and is recommended for testing the dependence structure between a small number of assets. The second test may not be consistent against all alternatives but it requires kernel estimation of only a univariate density function, and hence is useful for testing the dependence structure between a large number of assets. We justify our tests for both observable multivariate strictly stationary time series and for standardized innovations of GARCH models. A simulation study demonstrates the efficacy of both tests. When applied to equity return data and exchange rate return data, we find strong evidence against the normal copula, but little evidence against the more flexible Student's $t$ copula.
\end{abstract}

Keywords: Copulas, correlation, Normal copula, Student's t copula, nonlinear comovements, goodness-of-fit tests, GARCH.

JEL: C52, C32, C14.

\footnotetext{
${ }^{*}$ The authors would like to thank Rob Engle, Nour Meddahi, seminar participants at Rochester, Texas A\&M, and conference participants at 2003 NBER/NSF Time Series Conference in Chicago, C.R.E.D.I.T. 2003 International Conference on Dependence Modelling for Credit Portfolios in Venice, Italy for helpful comments. The authors gratefully acknowledge financial support from NSF/USA (Chen and Fan) and the IAM Programme in Hedge Fund Research at LSE (Patton). Contact information: Chen: Department of Economics, New York University, 269 Mercer Street, New York, NY 10003, USA. Email: xiaohong.chen@nyu.edu; Fan: Department of Economics, Vanderbilt University, Nashville TN 37235-1819, USA. Email: yanqin.fan@vanderbilt.edu; Patton: Financial Markets Group, London School of Economics, Houghton Street, London WC2A 2AE, UK. Email: a.patton@lse.ac.uk.
} 


\section{Introduction}

The dependence between returns on risky assets can have a profound impact on portfolio and risk management decisions. The dependence structure between the assets, in addition to the expected return and risk of the individual assets, will affect the distribution of portfolio returns and thus the utility that investors derive from a particular investment decision. Economic theory sheds little light on the dependence structures to expect between financial assets, and so finding a good specification is essentially an empirical problem. The most widely used dependence model is that of multivariate normality, making linear correlation a sufficient statistic for the dependence structure. However evidence that equity returns have a dependence structure that is not consistent with multivariate normality, or even multivariate ellipticity more generally, has recently been presented by Longin and Solnik (2001) and Ang and Chen (2002). Equity returns appear to be more correlated during downturns than during upturns. Similar evidence for the dependence between exchange rates was reported in Patton (2002a).

The evidence reported in these papers has prompted research on flexible models of dependence between risky assets. One such line of research employs copulas to model the possibly nonlinear contemporaneous dependence structure of multivariate financial time series. Numerous applications of copula models have appeared in recent work, on multivariate option pricing; portfolio and market Value-at-Risk calculations; default and credit risk correlations; contagion; tail-dependence; and time-varying contemporaneous conditional dependence between different assets, for example. See Frees and Valdez (1998), Embrechts et al. (2002) and Bouyé et al. (2000) for reviews of copula methods in finance and insurance. ${ }^{1}$ The main reason for the increasing popularity and success of copulas in modeling multivariate financial time series is its flexibility in separately modeling the marginal behavior of each individual series and the dependence structure between individual series. The theoretical foundation is provided by Sklar's (1959) theorem; any multivariate distribution with continuous marginals can be uniquely decomposed into a copula function and its univariate marginals, where a copula function is simply a multivariate distribution function with uniform marginals. One important property of copulas and copula-based measures of dependence is their invariance to any increasing transformation of the original individual time series. As a result the copula approach can flexibly model nonlinear and asymmetric dependence among multiple time series often found in financial series without the influence of the marginal distribution of each individual series.

In most of the existing work on modeling the dependence structure of multivariate financial time

\footnotetext{
${ }^{1}$ A non-exhaustive list of references include Hull and White (1998), Rosenberg (2003), Costinot, et al. (2000), Li (2000), Frey and McNeil (2001), Schonbucher and Schubert (2001), Cherubini and Luciano (2001, 2002), Duan (2002), Hu (2002), Junker and May (2002), Mashal and Zeevi (2002), Patton (2002a, b, 2004), Rockinger and Jondeau (2002), Dias and Embrechts (2003), Embrechts, et al. (2003) and Breymann, et al (2003).
} 
series via the copula approach, parametric copulas are used to model the contemporaneous dependence structure between univariate time $\operatorname{series}^{2}$ or between innovations of univariate parametric time series models. Commonly used parametric copulas include the normal (or Gaussian) copula, the Student's $t$ copula, Frank copula, Clayton copula and Gumbel copula. ${ }^{3}$ As these parametric copulas lead to models that may have completely different dependence properties, it is important in any empirical application to check whether the chosen parametric copula correctly specifies the dependence structure of the multivariate time series regardless of the marginal distributions of individual series.

Recently some authors have applied classical statistical tests such as the $\chi^{2}$ and the AndersonDarling tests of a parametric copula specification to financial data. For example, Breymann, et al (2003) test various parametric specifications of the copula of multivariate high-frequency data, while Junker and May (2002) apply these tests to testing parametric specifications of the copula of a set of risk factors that determine a portfolio. In both papers, the marginal distributions of the univariate time series are unspecified, and should be treated as infinite-dimensional unknown nuisance parameters. As a result, the existing critical values for the classical Anderson-Darling and the $\chi^{2}$ tests are no longer valid. This problem is also noted by Klugman and Parsa (1999) and Malevergne and Sornette (2003). In their test for the normal copula for multivariate i.i.d. data, Malevergne and Sornette (2003) have recommended the use of bootstrap to compute empirical critical values of the Anderson-Darling test. However, it is difficult to implement bootstrap for general financial multivariate time series models.

In this paper, we propose two simple and asymptotically valid tests of the goodness-of-fit of any parametric copula model of the contemporaneous dependence structure between multiple time series. Both tests are based on the multivariate probability integral transform and the kernel smoothing approach, hence are distribution-free and are easy to compute. ${ }^{4}$ The first one is a consistent test that requires the kernel estimation of a multivariate density function and is recommended for testing the dependence structure between a small number of financial time series; the second one may not be consistent against all alternatives, but it only requires kernel estimation of a univariate density function, and hence is very useful for testing the dependence structure between a large number of time series. We justify the proposed tests for both observable multivariate strictly stationary time series and for standardized innovations of GARCH models. Simulation results demonstrate that both tests are conservative for realistic sample sizes and data generating processes. Nevertheless, both tests perform well in terms of power. In view of the different power

\footnotetext{
${ }^{2}$ There are also a few papers using the copula approach to modeling (nonlinear) temporal dependence of individual time series, see Chen and Fan (2002), Gagliardini and Gourieroux (2002), and the references therein.

${ }^{3}$ Due to the well-known "curse of dimensionality" problem, fully nonparametric copula modeling, although theoretically feasible (see e.g. Fermanian and Scaillet, 2002), is practically difficult to implement when the number of series being modeled is greater than three.

${ }^{4}$ Matlab code for each test is available on the web, see http://fmg.lse.ac.uk/ patton/code.html.
} 
properties of smoothing-based tests and non-smoothing tests, our proposed tests of the goodnessof-fit of a parametric copula model complement the Anderson-Darling test and $\chi^{2}$ test already used in the copula literature.

We apply our proposed tests to determining whether the dependence structure of up to 30 major U.S. equities and up to 20 major exchange rates can be adequately described using the normal (or Gaussian) copula. Recently, the normal copula has been applied to model multivariate financial series as a way to relax the multivariate normal distribution assumption yet still retain computational simplicity. For example, Hull and White (1998) applied the normal copula in portfolio value-at-risk calculation, and Duan (2002) employed the normal copula in option pricing. Due to its wide practical use in finance, a researcher should not abandon a normal copula model without reasonable evidence against it. Our findings are as follows: For U.S. equity returns and for their standardized GARCH residuals, it is difficult to reject normal copula hypothesis in bivariate analyses; however, as the collection of assets gets larger (moving from 2 to 5, 10 and 30 assets) the normal copula provides a poorer and poorer fit. For exchange rates and for their standardized GARCH residuals, we can reject normal copula hypothesis even in bivariate analyses.

Since the normal copula implies no tail dependence nor 'asymmetric correlation' among different financial assets, it is not too surprising that we find evidence against normal copula in multivariate equity returns and exchange rates. Recently, Breymann et al. (2003) and Mashal and Zeevi (2002) have recommended the more flexible Student's t-copula model. Interestingly, our tests of the Student's t-copula hypothesis suggest that it is a reasonable model of the dependence structure between equity returns and between exchange rates. Our findings indicate that non-normalities in the dependence structure of equity returns and exchange rates, such as tail dependence, should be incorporated into multivariate modelling of these series.

The rest of this paper is organized as follows. Section 2 briefly reviews some facts on copulas. In Section 3, we introduce the two test statistics and establish their null limiting distributions for observable multivariate strictly stationary time series. Section 4 extends both tests to residuals of a multivariate time series model in which each individual time series is modeled by a GARCH model and their contemporaneous dependence is characterized by a parametric copula. In Section 5 the results of a Monte Carlo study of the finite sample size and power of the tests are reported. Section 6 presents analyses of the tenability of the normal copula and Student's t copula as models for the dependence structure of collections of daily U.S. equity returns, ranging from 2 assets to all 30 stocks in the Dow Jones Industrial Average. Section 7 presents an equivalent analysis for 20 major exchange rates. Section 8 briefly concludes. Regularity conditions and a sketch of proofs are collected in the Appendix. 


\section{A Brief Review of Copulas}

Suppose we observe a multivariate time series $\left\{\mathbf{Y}_{t} \equiv\left(Y_{1 t}, \ldots, Y_{d t}\right)\right\}_{t=1}^{n}$ from a multivariate strictly stationary ergodic process whose invariant distribution is the same as the probability distribution of the multivariate random variable $\mathbf{Y} \equiv\left(Y_{1}, \ldots, Y_{d}\right)$. We assume that $\left(Y_{1}, \ldots, Y_{d}\right)$ has a continuous joint distribution $H\left(y_{1}, \ldots, y_{d}\right)$ over $\Pi_{j=1}^{d} \mathcal{Y}_{j} \subseteq \mathcal{R}^{d}$, and continuous marginal distributions $F_{j}\left(y_{j}\right)$ for $j=1, \ldots, d$. By Sklar's (1959) theorem, there exists a unique copula function $C\left(u_{1}, \ldots, u_{d}\right) \equiv$ $H\left(F_{1}^{-1}\left(u_{1}\right), \ldots, F_{d}^{-1}\left(u_{d}\right)\right)$ associated with the joint distribution $H\left(y_{1}, \ldots, y_{d}\right)$, where $0 \leq u_{j} \leq$ 1 for $j=1, \ldots, d$. Let $U_{j t} \equiv F_{j}\left(Y_{j t}\right)$ and $U_{j} \equiv F_{j}\left(Y_{j}\right)$ for $j=1, \ldots, d$. Then by definition the multivariate time series $\left\{\mathbf{U}_{t} \equiv\left(U_{1 t}, \ldots, U_{d t}\right)\right\}_{t=1}^{n}$ is again a strictly stationary process whose invariant distribution is the same as the probability distribution of $\mathbf{U} \equiv\left(U_{1}, \ldots, U_{d}\right)$, which is simply the copula function $C\left(u_{1}, \ldots, u_{d}\right)$ with $0 \leq u_{j} \leq 1$ for $j=1, \ldots, d$.

Throughout the paper, we shall consider two popular copula models: the multivariate normal copula and the multivariate Student's t-copula:

The normal copula: the $d$-dimensional normal (or Gaussian) copula is derived from the $d$ - dimensional Gaussian distribution. Let $\Phi$ denote the scalar standard normal distribution, and $\Phi_{\Sigma, d}$ the $d$-dimensional normal distribution with correlation matrix $\Sigma$. Then the $d$-dimensional normal copula with correlation matrix $\Sigma$ is

$$
C(\mathbf{u} ; \Sigma)=\Phi_{\Sigma, d}\left(\Phi^{-1}\left(u_{1}\right), \ldots, \Phi^{-1}\left(u_{d}\right)\right)
$$

whose copula density is

$$
c(\mathbf{u} ; \Sigma)=\frac{1}{\sqrt{\operatorname{det}(\Sigma)}} \exp \left\{-\frac{\left(\Phi^{-1}\left(u_{1}\right), \ldots, \Phi^{-1}\left(u_{d}\right)\right)^{\prime}\left(\Sigma^{-1}-I_{d}\right)\left(\Phi^{-1}\left(u_{1}\right), \ldots, \Phi^{-1}\left(u_{d}\right)\right)}{2}\right\} .
$$

The Student's t-copula: the $d$ - dimensional (standardized) Student's t-copula is derived from the $d$-dimensional standardized Student's t-distribution. ${ }^{5}$ Let $T_{\nu}$ be the scalar standardized Student's t distribution with $\nu>2$ degrees of freedom, and $T_{\Sigma, \nu}$ be the $d$-dimensional standardized Student's t distribution with $\nu>2$ degrees of freedom and a correlation matrix $\Sigma$. Then the $d$-dimensional (standardized) Student's t-copula with correlation matrix $\Sigma$ is

$$
C(\mathbf{u} ; \Sigma, \nu)=T_{\Sigma, \nu}\left(T_{\nu}^{-1}\left(u_{1}\right), \ldots, T_{\nu}^{-1}\left(u_{d}\right)\right),
$$

where $T_{\nu}^{-1}\left(u_{1}\right)$ is the inverse of the scalar standardized Student's t distribution function $T_{\nu}$. The

\footnotetext{
${ }^{5}$ For interpretability we work with the standardized Student's t copula and distribution here, rather than the nonstandardized Student's t copula and distribution. The difference is that we constrain $\nu>2$, and define the matrix $\Sigma$ to be the correlation matrix. In the non-standardized case the matrix $\Sigma$ is a 'scale' matrix and the covariance matrix, if it exists (i.e., if $\nu>2$ ) is equal to $\Sigma \nu /(\nu-2)$. We will refer to the standardized Student's t copula as simply the Student's t copula for the remainder of the paper.
} 
(standardized) Student's $t$ copula density is:

$$
\begin{aligned}
c(\mathbf{u} ; \Sigma, \nu) & =\frac{\Gamma\left(\frac{\nu+d}{2}\right)\left[\Gamma\left(\frac{\nu}{2}\right)\right]^{d-1}}{\sqrt{\operatorname{det}(\Sigma)}\left[\Gamma\left(\frac{\nu+1}{2}\right)\right]^{d}}\left(1+\frac{\mathbf{x}^{\prime} \Sigma^{-1} \mathbf{x}}{\nu-2}\right)_{i=1}^{-\frac{\nu+d}{2}} d\left(1+\frac{x_{i}^{2}}{\nu-2}\right)^{\frac{\nu+1}{2}} \\
\text { where } \mathbf{x} & =\left(x_{1}, \ldots, x_{d}\right)^{\prime}, \quad x_{i}=T_{\nu}^{-1}\left(u_{i}\right) .
\end{aligned}
$$

Just as the univariate Student's $t$ distribution generalizes the normal distribution to allow for fat tails, the Student's $t$ copula generalizes the normal copula to allow for joint fat tails, i.e., an increased probability of joint extreme events.

Tail dependence: The "tail dependence" is a key dependence measure to consider when comparing the normal and Student's $t$ copulas, see Joe (1997). This is a measure of dependence between random variables in the extreme upper and lower joint tails. For example with $d=2$, if we assume that $Y_{1 t} \sim F_{1}$ and $Y_{2 t} \sim F_{2}$ with a bivariate copula function $C$, and define $U_{1 t}=F_{1}\left(Y_{1 t}\right)$ and $U_{2 t}=F_{2}\left(Y_{2 t}\right)$, then the coefficients of upper and lower tail dependence are defined as:

$$
\begin{aligned}
\tau^{L} & =\lim _{q \rightarrow 0} \operatorname{Pr}\left[U_{1 t} \leq q \mid U_{2 t} \leq q\right]=\lim _{q \rightarrow 0} \frac{C(q, q)}{q} \\
\tau^{U} & =\lim _{q \rightarrow 1} \operatorname{Pr}\left[U_{1 t}>q \mid U_{2 t}>q\right]=\lim _{q \rightarrow 1} \frac{1-2 q+C(q, q)}{1-q}
\end{aligned}
$$

Informally, the coefficients of upper (lower) tail dependence measure the probability of an extremely large positive (negative) return on one asset given that the other asset has yielded an extremely large positive (negative) return. If the two assets have a bivariate normal copula, then both upper and lower tail dependence coefficients are zero, i.e., the bivariate normal copula generates zero tail dependence. ${ }^{6}$ The Student's $t$ copula, on the other hand, generates positive tail dependence even if the correlation coefficient is zero. The Student's $t$ copula is symmetric and so the upper and lower tail dependence coefficients for this copula are equal. The tail dependence coefficients for the bivariate Student's $t$ copula with degrees of freedom $\nu>2$ and correlation coefficient $\rho$ are:

$$
\tau^{L}=\tau^{U}=2 T_{\nu+1}\left(-\sqrt{\nu-1} \sqrt{\frac{1-\rho}{1+\rho}}\right),
$$

see Embrechts et al (2002). The tail dependence coefficients of a bivariate $t$-copula for a few different choices of $\rho$ and $\nu$ are given in Table 1 .

\section{[ INSERT TABLE 1 ABOUT HERE ]}

While the above table is useful for distinguishing between the bivariate normal and t copulas, it does not give us an idea of how "different" the normal and t copulas are as a function of the dimension of the copula. In our empirical application in Section 6 we find quite different results

\footnotetext{
${ }^{6}$ However, this does not mean that the bivariate normal copula (with correlation coefficient $\rho$ ) goes to the independent copula $u_{1} u_{2}$ unless $\rho=0$. More generally, tail independence (i.e., $\tau^{L}=0, \tau^{U}=0$ ) is not equivalent to independence in the tail (i.e., $\left.\lim _{y_{1}, y_{2}}\left\{H\left(y_{1}, y_{2}\right) /\left[F_{1}\left(y_{1}\right) F_{2}\left(y_{2}\right)\right]\right\}=1\right)$, see e.g. Malevergne and Sornette (2003).
} 
as the dimension of the copula under analysis is increased. In Table 2 we use the Kullback-Leibler Information Criterion (KLIC) to measure the distance between the normal and $\mathrm{t}$ copulas, as a function of the dimension and the degrees of freedom parameter, for correlation of 0.5. This table confirms that for any given dimension, as the degrees of freedom parameter increases the KLIC distance gets smaller. It also indicates that for any given degrees of freedom, as the dimension of the copula increases the KLIC distance gets larger, and the impact of the dimension is substantial. For example, the distance between the bivariate normal and $t_{10}$ copulas is less than the distance between the 30 dimensional normal and $t_{50}$ copulas. For univariate densities most people substitute the normal density for a Student's $t$ density with degrees of freedom greater than 30; Table 2 shows that such a substitution may not be appropriate for higher dimension cases.

\section{[ INSERT TABLE 2 ABOUT HERE ]}

\section{Two Tests of a Parametric Copula for Time Series Data}

Let $\left\{C_{0}\left(u_{1}, \ldots, u_{d} ; \alpha\right): \alpha \in \mathcal{A}\right\}$ be a class of parametric copulas for $\mathbf{Y} \equiv\left(Y_{1}, \ldots, Y_{d}\right)$. We are interested in testing the null hypothesis:

$$
H_{0}: \operatorname{Pr}\left(C\left(U_{1}, \ldots, U_{d}\right)=C_{0}\left(U_{1}, \ldots, U_{d} ; \alpha_{0}\right)\right)=1 \text { for some } \alpha_{0} \in \mathcal{A}
$$

against the alternative hypothesis:

$$
H_{1}: \operatorname{Pr}\left(C\left(U_{1}, \ldots, U_{d}\right)=C_{0}\left(U_{1}, \ldots, U_{d} ; \alpha\right)\right)<1 \text { for all } \alpha \in \mathcal{A} \text {. }
$$

Let $C_{0 j}\left(u_{1}, \ldots, u_{j} ; \alpha_{0}\right)$ denote the joint distribution function of $U_{1}, \ldots, U_{j}$ under $H_{0}$. It is given by

$$
C_{0 j}\left(u_{1}, \ldots, u_{j} ; \alpha_{0}\right)=C_{0}\left(u_{1}, \ldots, u_{j}, 1, \ldots, 1 ; \alpha_{0}\right) .
$$

In addition, let $C_{0 j}\left(U_{j} ; \alpha_{0} \mid U_{1}, \ldots, U_{j-1}\right)$ denote the conditional distribution function of $U_{j}$ given $\left(U_{j-1}, \ldots, U_{1}\right)$ under $H_{0}$. It can be derived via

$$
C_{0 j}\left(u_{j} ; \alpha_{0} \mid u_{1}, \ldots, u_{j-1}\right)=\frac{\partial^{j-1} C_{0 j}\left(u_{1}, \ldots, u_{j} ; \alpha_{0}\right)}{\partial u_{1} \cdot \ldots \cdot \partial u_{j-1}} / \frac{\partial^{j-1} C_{0, j-1}\left(u_{1}, \ldots, u_{j-1} ; \alpha_{0}\right)}{\partial u_{1} \cdot \ldots \cdot \partial u_{j-1}} .
$$

Define the following probability integral transform ${ }^{7}$

$$
Z_{1} \equiv U_{1}, \quad Z_{j} \equiv C_{0 j}\left(U_{j} ; \alpha_{0} \mid U_{1}, \ldots, U_{j-1}\right) \text { for } j=2, \ldots, d .
$$

\footnotetext{
${ }^{7}$ This probability integral transform was first introduced by Rosenblatt (1952), and has been used recently to evaluate density forecasts in Diebold, et al (1998), to test parametric conditional distributions of dynamic regression models in Bai (2003) and Corradi and Swanson (2001), and to test goodness of fit of conditional distributions of diffusion models in Thompson (2002) and Hong and Li (2002).
} 


\subsection{A consistent test}

As the copula function is a multivariate distribution function, it follows from Rosenblatt (1952) that $H_{0}$ holds if and only if the probability integral transformed random variables $Z_{1}, \ldots, Z_{d}$ are independent and identically distributed as a Uniform $[0,1]$ random variable. Hence, $H_{0}$ is equivalent to

$$
H_{0}^{\prime}: P\left(g\left(Z_{1}, \ldots, Z_{d}\right)=1\right)=1,
$$

where $g\left(z_{1}, \ldots, z_{d}\right)$ is the joint density function of $Z_{1}, \ldots, Z_{d}$.

To test $H_{0}^{\prime}$, we need to first estimate the joint density function $g\left(z_{1}, \ldots, z_{d}\right)$. For each $t=1, \ldots, n$, we denote

$$
Z_{1 t}=U_{1 t}, \quad Z_{j t}=C_{0 j}\left(U_{j t} ; \alpha_{0} \mid U_{1 t}, \ldots, U_{j-1, t}\right) \text { for } j=2, \ldots, d .
$$

Then $\left\{\mathbf{Z}_{t} \equiv\left(Z_{1 t}, \ldots, Z_{d t}\right)\right\}_{t=1}^{n}$ becomes an infeasible time series sample of $\mathbf{Z} \equiv\left(Z_{1}, \ldots, Z_{d}\right)$, which can be used to construct an infeasible kernel estimator $\bar{g}\left(z_{1}, \ldots, z_{d}\right)$ of $g\left(z_{1}, \ldots, z_{d}\right)$ :

$$
\bar{g}\left(z_{1}, \ldots, z_{d}\right)=\frac{1}{n h^{d}} \sum_{t=1}^{n}\left[\Pi_{j=1}^{d} K_{h}\left(z_{j}, Z_{j t}\right)\right],
$$

and one could then test $H_{0}$ or $H_{0}^{\prime}$ based on

$$
\bar{I}_{n}=\int_{0}^{1} \ldots \int_{0}^{1}\left[\bar{g}\left(z_{1}, \ldots, z_{d}\right)-1\right]^{2} d z_{1} \ldots d z_{d}
$$

where $h \equiv h_{n}$ is a bandwidth goes to zero, and $K_{h}(x, y)$ is a univariate boundary kernel defined as

$$
K_{h}(x, y)= \begin{cases}k\left(\frac{x-y}{h}\right) / \int_{-(x / h)}^{1} k(u) d u, & \text { if } x \in[0, h), \\ k\left(\frac{x-y}{h}\right), & \text { if } x \in[h, 1-h], \\ k\left(\frac{x-y}{h}\right) / \int_{-1}^{(1-x) / h} k(u) d u, & \text { if } x \in(1-h, 1],\end{cases}
$$

in which $k(\cdot)$ is a symmetric univariate second order kernel function supported on $[-1,1]$. The above boundary kernel is used in Hong and Li (2002). Müller (1991) provides general optimal boundary kernels for pointwise kernel estimation, while Fan and Rilstone (2001) employ the general boundary kernels in testing the parametric specification of a hazard function.

Of course the time series $\left\{\mathbf{Z}_{t} \equiv\left(Z_{1 t}, \ldots, Z_{d t}\right)\right\}_{t=1}^{n}$ is not observable since $F_{j}, j=1, \ldots, d$ and $\alpha_{0}$ are unknown, hence $\bar{g}\left(z_{1}, \ldots, z_{d}\right)$ and $\bar{I}_{n}$ cannot be computed. Fortunately we can compute pseudo observations on $\mathbf{Z} \equiv\left(Z_{1}, \ldots, Z_{d}\right)$ as follows:

$$
\hat{Z}_{1 t}=\hat{F}_{1}\left(Y_{1 t}\right), \quad \hat{Z}_{j t}=C_{0 j}\left(\hat{F}_{j}\left(Y_{j t}\right) ; \hat{\alpha} \mid \hat{F}_{1}\left(Y_{1 t}\right), \ldots, \hat{F}_{j-1}\left(Y_{j-1, t}\right)\right),
$$

where $j=2, \ldots, d, t=1, \ldots, n, \hat{\alpha}$ is a $\sqrt{n}$-consistent estimator of $\alpha_{0}$ under the null hypothesis, and $\hat{F}_{j}(y)$ is the rescaled empirical distribution function of $F_{j}(y)$ :

$$
\hat{F}_{j}\left(y_{j}\right)=\frac{1}{n+1} \sum_{t=1}^{n} 1\left(Y_{j t} \leq y_{j}\right) .
$$


As in Fan (1994) and Hong and Li (2002), we can now base our test on

$$
\widehat{I}_{n}=\int_{0}^{1} \ldots \int_{0}^{1}\left[\hat{g}\left(z_{1}, \ldots, z_{d}\right)-1\right]^{2} d z_{1} \ldots d z_{d}
$$

where $\hat{g}\left(z_{1}, \ldots, z_{d}\right)$ is the kernel estimator of $g\left(z_{1}, \ldots, z_{d}\right)$ constructed from the pseudo observations $\left\{\widehat{\mathbf{Z}}_{t} \equiv\left(\widehat{Z}_{1 t}, \ldots, \widehat{Z}_{d t}\right)\right\}_{t=1}^{n}$ as follows:

$$
\hat{g}\left(z_{1}, \ldots, z_{d}\right)=\frac{1}{n h^{d}} \sum_{t=1}^{n}\left[\Pi_{j=1}^{d} K_{h}\left(z_{j}, \hat{Z}_{j t}\right)\right] .
$$

The asymptotic null distribution of $\widehat{I}_{n}$ will be derived in two steps: In the first step, we establish the asymptotic null distribution of $\bar{I}_{n}$; in the second step, we show that the asymptotic null distribution of $\widehat{I}_{n}$ is the same as that of $\bar{I}_{n}$.

To establish the asymptotic null distribution of $\bar{I}_{n}$, we follow Fan and Ullah (1999). In the following we assume that the observed multivariate time series $\left\{\mathbf{Y}_{t} \equiv\left(Y_{1 t}, \ldots, Y_{d t}\right)\right\}_{t=1}^{n}$ is strictly stationary and absolutely regular (or beta-mixing), see the appendix for the definition of an absolutely regular process. One advantage of this assumption is that the probability integral transformed time series $\left\{\mathbf{Z}_{t} \equiv\left(Z_{1 t}, \ldots, Z_{d t}\right)\right\}_{t=1}^{n}$ will also be stationary and absolutely regular, with the same decay rate as that of $\left\{\mathbf{Y}_{t} \equiv\left(Y_{1 t}, \ldots, Y_{d t}\right)\right\}_{t=1}^{n}$. This will allow us to test the correct specification of the contemporaneous dependence structure among $Y_{1 t}, \ldots, Y_{d t}$ (for arbitrarily fixed $t$ ), while leaving the temporal persistence structure of $\left\{\mathbf{Y}_{t} \equiv\left(Y_{1 t}, \ldots, Y_{d t}\right)\right\}_{t=1}^{n}$ to be totally unspecified as long as it is stationary and absolutely regular.

THEOREM 3.1 Under (C1)-(C3) and (A1)-(A2) stated in the Appendix, $T_{n d} \equiv\left(n h^{d / 2} \widehat{I}_{n}-\right.$ $\left.c_{d n}\right) / \sigma_{d} \rightarrow N(0,1)$ in distribution under $H_{0}$, where

$$
\begin{aligned}
c_{d n} & =h^{d / 2}\left[\left(h^{-1}-2\right) \int_{-1}^{1} k^{2}(w) d w+2 \int_{0}^{1} \int_{-1}^{z} k_{z}^{2}(y) d y d z\right]^{d} \\
\sigma_{d}^{2} & =2\left\{\int_{-1}^{1}\left[\int_{-1}^{1} k(u+v) k(v) d v\right]^{2 d} d u\right\}^{d}
\end{aligned}
$$

where $k_{z}(y)=k(y) / \int_{-1}^{z} k(u) d u$.

Remark: Although in Theorem 3.1 we assume that the same bandwidth $h$ is used in all directions, one can easily extend the result to the case where a different bandwidth is used in each different direction:

$$
\begin{aligned}
T_{n d}^{*} & =\frac{n \Pi_{i=1}^{d} h_{i}^{1 / 2} \hat{I}_{n}-c_{d n}^{*}}{\sigma_{d}} \\
c_{d n}^{*} & =\Pi_{i=1}^{d}\left\{h_{i}^{1 / 2}\left[\left(h_{i}^{-1}-2\right) \int_{-1}^{1} k^{2}(w) d w+2 \int_{0}^{1} \int_{-1}^{z} k_{z}^{2}(y) d y d z\right]\right\} .
\end{aligned}
$$


Note that the asymptotic distribution of $\widehat{I}_{n}$ under the null hypothesis has the same variance as that of the bias-corrected test in Fan (1994), but a different center term caused by the use of the boundary kernel. The derivation of the center term is relegated to the Appendix.

Theorem 3.1 suggests the following simple, distribution-free test for the correct specification of a parametric copula $\left\{C_{0}\left(u_{1}, \ldots, u_{d} ; \alpha\right): \alpha \in \mathcal{A}\right\}$ : reject $H_{0}$ at a given significance level $5 \%$ (say), if $T_{n d}>z_{5 \%}$, where $z_{5 \%}$ is the upper $5 \%$ quantile of the standard normal distribution. Following Fan (1994) or Fan and Ullah (1999), one can show that this test is consistent against $H_{1}$.

One may also obtain a $\chi^{2}$ test from Theorem 3.1 by noting that $T_{n d}^{2} \rightarrow \chi_{[1]}^{2}$ in distribution under $H_{0}$. Compared with the normal test based on $T_{n d}$, the $\chi^{2}$ test based on $T_{n d}^{2}$ loses some power. However our simulation results reveal better size performance of the $\chi^{2}$ test $T_{n d}^{2}$ and hence we recommend it in practical applications.

Recently, we received a working paper by Fermanian (2003), who proposed to consistently test a parametric copula specification by nonparametric kernel estimation of the joint copula density of the original multivariate series. We note that Fermanian's test is closely related to the consistent test of the specification of a parametric density function in Fan (1994), while our first test is similar in spirit to the test in Hong and $\mathrm{Li}$ (2002) developed for testing the correct specification of univariate diffusion models. Hong and Li (2002) report Monte Carlo evidence that the consistent test based on the kernel density estimation of the probability integral transformed random variables has better size properties than the test based on the kernel density estimation of the original random variables, and we suspect that a similar conclusion may apply to the two consistent tests of parametric copula specifications: our test $T_{n d}$ and that in Fermanian (2003). In any case, for large dimensional multivariate time series models, neither our test based on $T_{n d}$ nor Fermanian's test will perform well. This motivates the second test in our paper.

\subsection{An Alternative Test of a High-Dimensional Copula Model}

It is well known that when the dimension $d$ is large, the kernel estimator may suffer from the "curse of dimensionality", which may affect the accuracy of the test proposed in the previous section. To circumvent this problem, we note that if $H_{0}$ holds, then the scalar random variable $W \equiv \sum_{j=1}^{d}\left[\Phi^{-1}\left(Z_{j}\right)\right]^{2}$ follows a $\chi_{[d]}^{2}$ distribution, where $\Phi^{-1}$ denotes the inverse of the standard normal distribution function. Breymann, et al (2003) apply the Anderson-Darling test to testing the distribution of $W$ or $H_{0}$ for multivariate high-frequency data. However, as $W$ is not observed, they use pseudo observations $\left\{\hat{W}_{t}\right\}_{t=1}^{n}$ defined below in implementing the Anderson-Darling test. It is known that parameter estimation will affect the critical values of the Anderson-Darling test in a complicated, non-trivial way. The conclusions may be erroneous if such effect is not taken into 
account. $^{8}$

In this section, we propose an alternative kernel-based test. Let $F_{\chi_{[d]}^{2}}(w)$ denote the distribution function of the $\chi_{[d]}^{2}$ distribution and $g_{W}(\cdot)$ its density function. Noting that $F_{\chi_{[d]}^{2}}(W)$ follows the uniform distribution on the interval $[0,1]$, we develop a test by comparing a kernel estimate of $g_{W}(\cdot)$ with the uniform density. In particular, the kernel estimate of $g_{W}(w)$ is defined as

$$
\hat{g}_{W}(w)=\frac{1}{n h} \sum_{t=1}^{n} K_{h}\left(w, F_{\chi_{[d]}^{2}}\left(\hat{W}_{t}\right)\right),
$$

where

$$
\hat{W}_{t}=\sum_{j=1}^{d}\left[\Phi^{-1}\left(\hat{Z}_{j t}\right)\right]^{2} .
$$

The alternative test is based on

$$
\widehat{J}_{n}=\int_{0}^{1}\left[\hat{g}_{W}(w)-1\right]^{2} d w .
$$

THEOREM 3.2 Under similar conditions to those in Theorem 3.1, $T_{n 1} \equiv\left(n h^{1 / 2} \widehat{J}_{n}-c_{1 n}\right) / \sigma_{1} \rightarrow$ $N(0,1)$ in distribution, where

$$
\begin{aligned}
c_{1 n} & =h^{1 / 2}\left[\left(h^{-1}-2\right) \int_{-1}^{1} k^{2}(w) d w+2 \int_{0}^{1} \int_{-1}^{z} k_{z}^{2}(y) d y d z\right] \\
\sigma_{1}^{2} & =2 \int_{-1}^{1}\left[\int_{-1}^{1} k(u+v) k(v) d v\right]^{2} d u .
\end{aligned}
$$

Compared with the previous test, the test $T_{n 1}$ only requires kernel density estimation in one dimension regardless of how large the true dimension of the multivariate series $d$ is. However, it may have trivial power against certain deviations from the null model.

\section{Testing a Parametric Copula for Residuals of Parametric Dy- namic Models}

In a number of empirical applications of copulas in modeling multivariate financial time series, univariate GARCH models are used first to model the individual time series and parametric copulas are then used to model the dependence structure of residuals from the univariate GARCH models. This class of multivariate time series models has proved to be successful in capturing the stylized

\footnotetext{
${ }^{8}$ Distribution-free versions of this test have been developed in the context of testing parametric regression models via the martingale transformation approach of Khmaladze (1981), see e.g. Bai (2003) and Stute, et al (1998) among others. However this approach can not be easily modified to test the parametric copula, as the marginal distribution functions are infinite-dimensional nuisance parameters in semiparametric copula models.
} 
facts of multivariate financial time series such as clusters of extreme values. The model takes the following form:

$$
\begin{aligned}
Y_{j t} & =X_{j t}^{\prime} \delta_{j}+\sigma_{j t} \epsilon_{j t}, \\
\sigma_{j t}^{2} & =\kappa_{j}+\beta_{j} \sigma_{j, t-1}^{2}+\gamma_{j}\left(Y_{j, t-1}-X_{j, t-1}^{\prime} \delta_{j}\right)^{2},
\end{aligned}
$$

where for each $j=1, \ldots, d,\left\{\epsilon_{j t}\right\}_{t=1}^{n}$ is i.i.d. with zero mean and unit variance and for each $t$, $\left(\epsilon_{1 t}, \ldots, \epsilon_{d t}\right)^{\prime}$ has copula $C\left(\epsilon_{1}, \ldots, \epsilon_{d}\right)$. The parameters of the GARCH models are assumed to satisfy

$$
\kappa_{j}>0, \beta_{j} \geq 0, \gamma_{j} \geq 0, \text { and } \beta_{j}+\gamma_{j} \leq 1, j=1, \ldots, d .
$$

This model and variants of it have been used in Patton (2002a), Rockinger and Jondeau (2002), and Junker and May (2002), to name only a few. Junker and May (2002) apply the above model with several parametric copulas to describe a set of risk factors that determine a portfolio. They use the $\chi^{2}$ test to check the validity of different parametric copulas including the Gaussian copula and the Students' $t$ copula. However, like the Anderson-Darling test, the correct critical values of the $\chi^{2}$ test for this model remain to be worked out.

In this section, we extend the tests proposed in the previous two sections to testing the correct specification of the parametric copulas for the residuals $\left(\epsilon_{1 t}, \ldots, \epsilon_{d t}\right)$.

The assumptions we adopt on the univariate GARCH models are the same as those in Bai (2002): For each $j=1, \ldots, d$, we assume the following conditions hold.

(G1) The $\epsilon_{j t}$ are i.i.d. with a cdf $F_{j}(x)$, which is continuous and strictly increasing. In addition, $\epsilon_{j t}$ is independent of $X_{j s}$ for $s \leq t$;

(G2) $E \sigma_{j t}^{2}<M_{j}$ and $E \epsilon_{j t}^{2}<M_{j}$ for all $t$ and for some $M_{j}<\infty$;

(G3) $n^{-1} \sum_{t=1}^{n} X_{j t} X_{j t}^{\prime}$ converges to a non-random and positive definite matrix;

(G4) There exists $\hat{\theta}_{j}$ such that $\sqrt{n}\left(\hat{\theta}_{j}-\theta_{j}\right)=O_{p}(1)$, where $\theta_{j}=\left(\delta_{j}^{\prime}, \kappa_{j}, \beta_{j}, \gamma_{j}\right)^{\prime}$.

An example of the estimator $\hat{\theta}_{j}$ satisfying the assumption (G4) is the QMLE of the univariate GARCH models, see Bollerslev and Wooldridge (1992), Robinson and Zaffaroni (2002) and the references therein for conditions under which the QMLE $\hat{\theta}_{j}$ satisfies $(\mathrm{G} 4)$. For each $j=1, \ldots, d$, given an initial value $\hat{\sigma}_{j 0}^{2}$ and the estimate $\hat{\theta}_{j}$, we compute the conditional variance via the following recursion:

$$
\hat{\sigma}_{j t}^{2}=\widehat{\kappa}_{j}+\hat{\beta}_{j} \hat{\sigma}_{j, t-1}^{2}+\hat{\gamma}_{j}\left(Y_{j, t-1}-\hat{\delta}_{j}^{\prime} X_{j, t-1}\right)^{2} .
$$

Define the residuals

$$
\hat{\epsilon}_{j t}=\left(Y_{j t}-\hat{\delta}_{j}^{\prime} X_{j t}\right) / \hat{\sigma}_{j t}, j=1, \ldots, d .
$$


Then we use $\left\{\left(\hat{\epsilon}_{1 t}, \ldots, \hat{\epsilon}_{d t}\right)\right\}_{t=1}^{n}$ to construct the test $T_{n d}^{\epsilon}$ as in Theorem 3.1 or the test $T_{n 1}^{\epsilon}$ as in Theorem 3.2.

THEOREM 4.1 Under conditions (G1)-(G4) and the same conditions as in Theorems 3.1 and 3.2 except the absolute regularity condition, Theorems 3.1 and 3.2 hold for $T_{n d}^{\epsilon}$ and $T_{n 1}^{\epsilon}$ respectively.

Theorem 4.1 implies that the use of QMLE of the parameters in the univariate GARCH models does not affect the asymptotic null distribution of the tests. This is due to the $\sqrt{n}$-consistency of the QMLE.

\section{A Simulation Study}

To assess the finite sample performance of the tests proposed above we conducted a series of Monte Carlo studies. We considered sample sizes $(n)$ of 500, 2500 and 5000, covering the various sizes likely to be encountered in empirical analyses of copulas of financial assets. Given the prevalence of the assumption of normality in finance, we focus on the ability of the proposed tests to reject the null hypothesis that the true copula is a normal copula. The data generating process (DGP) was such that the individual variables were conditionally normal, and that the copula was some mixture of the Normal copula and the Student's $t$ copula with 4 degrees of freedom. We let the mixing parameter, $p$, vary between zero and one. The DGP copula is Normal and the null hypothesis is true when $p=0$, while the DGP copula is the Student's $t_{4}$ copula when $p=1$. The intermediate values of $p$ measure the power of the test to detect small deviations from the null. The DGP was specified to be:

$$
\left(U_{1 t}, \ldots, U_{d t}\right) \sim \operatorname{iid}(1-p) \cdot C_{\text {Normal }}(\rho)+p \cdot C_{t_{4}}(\rho), t=1,2, \ldots, n,
$$

where $C_{\text {Normal }}(\rho)$ is the $d$-dimensional normal copula with correlation matrix having value $\rho$ for its off-diagonal elements, and $C_{t_{4}}(\rho)$ is the $d$-dimensional Student's $t_{4}$ copula with correlation matrix having value $\rho$ for its off-diagonal elements. The coefficient $\rho$ is a measure of the correlation between the assets. We considered three values of $\rho$ to determine the power of the test for various levels of dependence: $\rho \in\{0.1,0.5,0.9\}$.

We considered two cases for the marginal distributions: the case that the individual variables were iid $\operatorname{Normal}(0,1)$; and the case that the individual variables have an $\mathrm{AR}(1)-\mathrm{GARCH}(1,1) \mathrm{DGP}$. That is,

$$
\begin{aligned}
Y_{i t}= & \Phi^{-1}\left(U_{i t}\right), i=1,2, \ldots, d \\
& \text { or } \\
Y_{i t}= & 0.01+0.05 Y_{i, t-1}+e_{i t}
\end{aligned}
$$




$$
\begin{aligned}
e_{i t} & =\sigma_{i t} \cdot \Phi^{-1}\left(U_{i t}\right) \\
\sigma_{i t}^{2} & =0.05+0.85 \sigma_{i, t-1}^{2}+0.1 e_{i, t-1}^{2}, i=1,2, \ldots, d
\end{aligned}
$$

where $\Phi^{-1}$ is the inverse of the scalar standard normal distribution function. In the first case there is no need to estimate parameters associated with the marginal distributions. In the second case the parameters of the $\operatorname{AR}(1)-G A R C H(1,1)$ model are estimated via QMLE.

We considered five values of the mixing parameter $p \in\{0,0.25,0.5,0.75,1\}$, and three values of the dimension of the assets $d \in\{2,5,30\}$. For the larger values of $d=5,30$, we only analyzed the performance of Test 2; as mentioned in the previous section Test 1 may have difficulties for high dimension problems. We used 500 Monte Carlo replications for each of the bivariate cases, and 100 replications for the higher dimension cases.

To test the null hypothesis of a normal copula, the $\hat{Z}_{i t}$ variables are computed as follows:

$$
\begin{aligned}
\operatorname{Let} \tilde{Y}_{i t} & =\Phi^{-1}\left(\hat{F}_{i}\left(Y_{i t}\right)\right) \\
\tilde{Y}_{t} & =\left[\tilde{Y}_{1 t}, \ldots, \tilde{Y}_{d t}\right]^{\prime} \\
\hat{Z}_{1 t} & =\hat{F}_{1}\left(Y_{1 t}\right) \\
\hat{Z}_{i t} & =\Phi\left(\frac{\tilde{Y}_{i t}-\tilde{Y}_{[1: i-1], t}^{\prime} \cdot \hat{\Sigma}_{[1: i-1,1: i-1]}^{-1} \cdot \hat{\Sigma}_{[1: i-1, i]}}{\sqrt{1-\hat{\Sigma}_{[i, 1: i-1]} \cdot \hat{\Sigma}_{[1: i-1,1: i-1]}^{-1} \cdot \hat{\Sigma}_{[1: i-1, i]}}}\right), i=2, \ldots, d
\end{aligned}
$$

where $\tilde{Y}_{[1: i-1], t}^{\prime}=\left[\tilde{Y}_{1 t}, \ldots, \tilde{Y}_{i-1, t}\right]$ and similarly for $\hat{\Sigma}_{[1: i-1, i]}$, and $\hat{\Sigma}_{[1: i-1,1: i-1]}^{-1} \equiv\left(\hat{\Sigma}_{[1: i-1,1: i-1]}\right)^{-1} \cdot \hat{\Sigma}$ is the estimate of the parameter of normal copula, which is simply the sample correlation matrix of $\tilde{Y}_{t} . \hat{F}_{i}$ is the estimated distribution function of $Y_{i t}$, consisting of parameters from the models for the mean and variance (if applicable) and the empirical distribution function of the estimated standardized residuals.

Throughout this simulation, and the empirical applications to follow, we employed the "quartic kernel":

$$
k(u)=\frac{15}{16}\left(1-u^{2}\right)^{2} \cdot \mathbf{1}\{|u| \leq 1\}
$$

which yields the following quantities necessary in the construction of the test statistics ${ }^{9}$ :

$$
\begin{aligned}
\int_{-x / h}^{1} k(u) d u & =\frac{1}{2}+\frac{15 x}{16 h}-\frac{5 x^{3}}{8 h^{3}}+\frac{3 x^{5}}{16 h^{5}} \\
\int_{-1}^{(1-x) / h} k(u) d u & =\frac{1}{2}+\frac{15(1-x)}{16 h}-\frac{5(1-x)^{3}}{8 h^{3}}+\frac{3(1-x)^{5}}{16 h^{5}} \\
\int_{-1}^{1} k^{2}(w) d w & =\frac{5}{7}
\end{aligned}
$$

\footnotetext{
${ }^{9}$ The final expression below was obtained using Mathematica.
} 


$$
\begin{aligned}
2\left\{\int_{-1}^{1}\left[\int_{-1}^{1} k(u+v) k(v) d v\right]^{2 d} d u\right\}^{d} & =2^{1-7 d}\left(\frac{3865}{49}\right)^{d} d^{d}, d=1,2, \ldots \\
\int_{0}^{1} \int_{-1}^{z} k_{z}^{2}(y) d y d z & =0.91985927266009985456
\end{aligned}
$$

The remaining input to the computation of the test statistics is the bandwidth, $h$. For Test 1 we allow the bandwidth to be different in each dimension, according to the rule-of-thumb $h_{i}=$ $V\left[\hat{Z}_{i, t}\right]^{1 / 2} n^{-1 /(d+4)}$. For Test 2 we use a similar rule-of-thumb, $h=V\left[\hat{W}_{t}\right]^{1 / 2} n^{-1 / 5}$. In both cases we use the two-sided test rather than the one-sided test, as it had better size properties in finite samples.

\section{[ INSERT TABLES 3 AND 4 ABOUT HERE ]}

The second column of Table 3 shows that in all cases Test 1 has reasonable empirical size, ranging from 0.000 to 0.056 when the nominal size is 0.05 . The test is too conservative in all but one case considered, but empirical size tends toward its nominal level as the sample size increases. Turning to the results on power, Table 3 suggests that Test 1 has reasonable power against the $t(4)$ copula for sample sizes 2500 and 5000 but may have difficulty detecting intermediate alternatives for sample sizes less than 5000. The degree of correlation does not seem to much affect the power of the test.

From Table 4 we also see that Test 2 has reasonable empirical size in this simulation: ranging from 0.000 to 0.040. Again, in all cases the empirical size increases towards its nominal level as the sample size increases. The power of Test 2 is comparable to that of Test 1 in this scenario. For the largest sample size both tests have reasonable power to reject the Normal copula.

\section{[ INSERT TABLES 5 AND 6 ABOUT HERE ]}

In Tables 5 and 6 we present the corresponding results to those in Tables 3 and 4 when the marginal distributions contain parameters that must be estimated before estimating the parameter of the copula. The marginal distributions are assumed to be $\mathrm{AR}(1)-\mathrm{GARCH}(1,1)$ processes with normal innovations, as in equations (5.3) to (5.5). The empirical size of both Test 1 and Test 2 is satisfactory in this case, and the power of both tests appears to be largely unaffected by the introduction of the five parameters per marginal distribution that must be estimated. This confirms the small-sample accuracy of the asymptotic theory in dealing with both parametric and nonparametric estimation error.

\section{[ INSERT TABLES 7 AND 8 ABOUT HERE ]}

In Tables 7 and 8 we investigate the performance of Test 2 to detect deviations from the normal copula when the dimension of the copula model is increased from 2 to 5 . Tables 7 and 8 suggest that deviations from normality of the form embodied by the Student's $t_{4}$ copula are well detected 
by Test 2. The empirical size of this test both with and without parametric estimation error in the marginal distributions is satisfactory, and the power of the test is much greater than in the bivariate case discussed above. For all levels of correlation the power is good, and particularly so for the two larger sample sizes. For sample sizes of 2500 and 5000 the smallest deviation from the null considered (when the DGP is a 75:25 mixture of the normal and the $t_{4}$ copula) is detected in almost all cases.

\section{[ INSERT TABLES 9 AND 10 ABOUT HERE ]}

In Tables 9 and 10 we repeat the analysis for the copula model of dimension 30 . This is very large relative to the types of models previously presented in the literature, but not large relative to the problems typically faced by portfolio managers and risk managers. We study this case to determine whether we may apply Test 2 to the study of large collections of risky assets in the next section. Tables 9 and 10 show that the size of the test is good for sample sizes of 2500 and 5000, but is somewhat over-sized for the case that the sample size is 500. This is not too surprising; one would not expect many people to estimate a 30-dimension density model with merely 500 observations at their disposal. The power of the test is very good in this case: it is equal to 1 for all the sample sizes and all the correlation levels considered, both with and without parametric estimation error in the marginal distributions.

\section{Testing Copula Hypotheses for US Equity Returns}

There is much accumulated evidence against the assumption of normal distribution for individual stock returns, dating back as far as Mills (1927). Such evidence is of course also sufficient to reject joint normality of stock returns. An interesting question, though, is whether the assumption of joint normality would be reasonable if we could somehow deal with non-normality in the distribution of individual returns. This is thus a question about the copula of these returns. Recently Malevergne and Sornette (2003) presented evidence that the bivariate normal copula hypothesis cannot be rejected for many pairs of equity returns. When moving to a larger collections of financial assets, Mashal and Zeevi (2002) find that a multivariate normal copula is more easily rejected in favor of a multivariate Student's $t$ copula. We note both of the papers conduct their respective tests under the assumption that the observed asset returns are i.i.d. over time, which is not be a good assumption for stock returns. In this section, we shall consider the goodness-of-fit tests of the normal copula and of the $t$-copula hypotheses for US equity returns without assuming that returns are i.i.d. over time.

We extend and generalize the work of Malevergne and Sornette (2003) to consider larger collections of assets (ranging from 2 to 30 assets) and to deal with the estimation error introduced by first employing conditional mean and variance filters for the individual returns. Further, we 
also test the goodness-of-fit of a normal copula with time-varying conditional correlations, and the goodness-of-fit of a Student's $t$ copula. Matlab code for testing each of these copula specifications is available, see footnote 4 .

We use daily returns on the 30 stocks in the Dow Jones Industrial Average over the period January 3, 1990 to December 31, 1999, yielding 2525 observations. We considered collections of assets ranging in dimension from 2 to 30 assets, to investigate the impact of the dimension of the multivariate model being considered on the plausibility of a normal copula and of a $t$-copula. For each dimension size, $d$, we randomly selected 100 distinct collections of $d$ assets from the 30 stocks. Analyzing all possible collections of $d$ assets was not feasible ${ }^{10}$ for most values of $d$. We randomly selected the collections of assets, rather than study collections of particular assets, so as to present results that may be taken as generally reflective of the plausibility of the normal copula (or of the $t$-copula) hypothesis for daily returns on liquid stocks. The results reported in this section may or may not be representative of those for infrequently traded stocks, or for emerging market stocks, and we leave the analysis of these alternative assets for future work.

\subsection{Testing the normal copula for US equity returns}

We considered the normal copula hypothesis in three 'forms'. The first is for the unconditional distribution of asset returns. That is, we transform the returns by their empirical distribution functions and test the normal copula hypothesis on these variables.

In the second case we recognize that daily equity returns exhibit serial correlation and volatility clustering, and we employ a simple $\operatorname{ARMA}(1,1)-\operatorname{GARCH}(1,1)$ filter to capture these. We then test the normality of the unconditional copula of the standardized returns. We will refer to this mean and variance filter simply as a GARCH filter below.

Finally, we consider the case that the conditional dependence between the returns may be timevarying. Some authors, see Tse (2000) and Engle (2002) amongst others, have reported that the conditional correlation between equity returns is not constant, and so analyzing the unconditional copula of asset returns or standardized asset returns is of less interest than analyzing the conditional copula. To allow for possible time variation in the conditional copula of these assets we employ the Dynamic Conditional Correlation (DCC) model of Engle (2002) and Engle and Sheppard (2001). This model allows the conditional correlation matrix of a collection of assets to be time-varying, and does so in a manner that easily extends to large collections of assets. This is important, as we wish to consider collections of up to 30 assets. We use the DCC model to parameterize the time variation in the correlation matrix of the normal copula. The conditional correlation matrix in a $\operatorname{DCC}(1,1)$ Normal copula model is given by:

$$
\Sigma_{t}=Q_{t}^{*-1} Q_{t} Q_{t}^{*-1}, \text { where }
$$

\footnotetext{
${ }^{10}$ For $d=15$, for example, the total number of combinations is ${ }^{30} C_{15}=155,117,520$.
} 


$$
\begin{aligned}
Q_{t}= & (1-\kappa-\beta) \bar{\Sigma}+\kappa \tilde{Y}_{t-1} \tilde{Y}_{t-1}^{\prime}+\beta Q_{t-1} \\
Q_{t}^{*}= & {\left[\begin{array}{cccc}
\sqrt{q_{11, t}} & 0 & \cdots & 0 \\
0 & \sqrt{q_{22, t}} & \cdots & 0 \\
\vdots & \vdots & \ddots & \vdots \\
0 & 0 & \cdots & \sqrt{q_{d d, t}}
\end{array}\right] }
\end{aligned}
$$

$q_{i i, t}$ is the $(i, i)^{t h}$ element of $Q_{t}$, and $\bar{\Sigma}$ is the sample correlation matrix of $\tilde{Y}_{t} \equiv \Phi^{-1}\left(\hat{F}\left(Y_{t}\right)\right)$. Thus allowing for dynamics of the form given by a $\operatorname{DCC}(1,1)$ model introduces two new parameters, $\kappa$ and $\beta$, that must be estimated.

Two points regarding our use of the DCC model should be made. The first is an acknowledgment that a rejection of the time-varying normal copula may be due either to the assumption of conditional normality of the copula, or to the particular specification for the dynamics of the conditional copula we employed. That is, it may be normality or the DCC model or both that generates a rejection. Flexible modeling of the correlation matrix of large collections of assets may be done in a number of ways; see Bauwens, et al. (2003) for a comprehensive survey of multivariate GARCH modeling,

A second, related, point that should be made is that a rejection of the conditional normal copula with DCC does not constitute evidence against the DCC model for correlations. The rejection of a conditional normal copula with DCC may suggest that correlations are not sufficient to characterize dependence between financial assets, and thus that we should employ other more general measures of dependence, but correlations themselves may be well modelled with the DCC model.

We firstly present the results of an analysis of the copula of randomly chosen pairs of assets from the Dow Jones 30. ${ }^{11}$ The correlation between the returns on these stocks ranged from 0.13 to 0.56 , and averages 0.26 across the 435 pairs of assets. We performed both Test 1 and Test 2 on these pairs of assets, and present the results in Table 11. This table shows that the bivariate normal copula is difficult to reject for these assets. In no case was the proportion of rejections greater than the $5 \%$ nominal size. The Monte Carlo results corresponding to this case, presented in Tables 3 and 4, suggest that the power of Tests 1 and 2 to detect deviations from the bivariate normal copula is reasonable only for "large" deviations, represented in the simulation as a copula that is wholly non-normal rather than a mixture of a normal and a non-normal copula. Overall these results indicate that for bivariate problems the normal copula may be an adequate description of U.S. equity return dependence structures, or that the deviations from normality in the bivariate case are not large. This finding is consistent with that of Malevergne and Sornette (2003), who also report that the normal copula is difficult to reject for pairs of equity returns.

\section{[ INSERT TABLE 11 ABOUT HERE ]}

\footnotetext{
${ }^{11}$ We do not report the parameter estimates for the ARMA, GARCH, or DCC models estimated prior to running the following tests as these are not of direct interest.
} 
In Table 12 we report the fraction of times that the normal copula was rejected for a collection of size $d$ of equities. All tests are conducted at the $5 \%$ level. We only conducted the second of the two tests proposed in the previous section.

\section{[ INSERT TABLE 12 ABOUT HERE ]}

Table 12 shows that as we increase the number of assets in the collection from 2 to 3 we see the fraction of rejections increases dramatically. In the first column, representing a test of the normality of the unconditional copula of these returns, the fraction of rejections goes from 0.03 to 0.59. In the second column, where we first employ a GARCH filter, the fraction goes from 0.01 to 0.17. In the final column, where we also allow for a time-varying conditional copula the fraction goes from 0.02 to 0.12 . Notice that allowing for time-varying conditional variance, and then for time-varying conditional variance and a time-varying conditional copula, decreases the fraction of rejections of normality. This suggests some of the rejections found in the first column may actually be due to time variation in the conditional distribution of these returns rather than non-normality.

As the number of assets in the collection increases we see that in all three cases the fraction of rejections increases, and that by the time this number reaches 10 the rejection frequency is $100 \%$ for all three cases. This stands in contrast to what one might have expected a priori. It would have been reasonable to expect that increasing the dimension of the copula being tested, without increasing the length of the time series being used, would have lead to a decrease in the power of the test to reject the null hypothesis, due to the increasing sparsity of data points in the support of the joint density. However we find the opposite: increasing the dimension of the problem improves the ability of the test to detect non-normality in our study. The simulation study results presented in Table 8 suggest that this is not due to a size distortion. Instead, Table 8 reported that the power of the test to detect weak non-normality (represented in the simulation study as a copula DGP that is mostly normal and only partially Student's $t_{4}$ copula) increased with the dimension of the copula under analysis, which is consistent with the results of our empirical study. This may be due to the greater KLIC distance between the normal and Student's $t$ copulas for higher dimensions, see Table 2.

Mashal and Zeevi (2002) similarly find that the normal copula is more easily rejected for larger collections of financial assets, though they considered testing the null of a normal copula against a Student's $t$ copula and assumed the observable multiple series are i.i.d., whereas we allow for a general alternative copula, for stationary multivariate serially dependent asset returns, and for preliminary filtering of the asset returns using parametric dynamic models of the conditional mean and variance.

The strong rejection of the conditional normal copula for collections of 5 or more U.S. equities is an important one. Bivariate problems are often studied in academic research, being taken as representative of higher dimension multivariate problems. However, practitioners are often 
concerned with larger collections of assets: portfolio managers generally have numerous assets in their portfolios, and risk managers have numerous exposures. Our results suggest that for bivariate problems the normal copula hypothesis is plausible, but for problems of higher dimensions the normal copula hypothesis is untenable. This suggests the need for more general methods of measuring and modeling the dependence between risky assets, and implies that portfolio managers and risk managers should be concerned about non-normality of the dependence structure between equity returns. To this end, we now turn to an analysis of the goodness of fit of the Student's $t$ copula.

\subsection{Testing the t-copula for US equity returns}

A natural generalization of the univariate normal distribution assumption for individual assets is the Student's $t$ distribution, which was first utilized in time series density modeling by Bollerslev (1987). Given the strong rejection of the normal copula it again seems natural to look to the Student's $t$ copula to provide a first step towards flexible copula modeling. As we already pointed out in Section 2, the Student's $t$ copula allows for joint extreme co-movements. However, it does not allow for asymmetries in the copula. Such asymmetries in equity return dependence structures have recently been reported in the finance literature, see Longin and Solnik (2001), Ang and Chen (2002) and Patton (2004), and so the Student's $t$ copula may also be too restrictive to provide a reasonable fit to equity data.

In order to implement the tests described above we must have an expression for computation

of the $\hat{Z}_{i t}$ variables. We do this by making use of a property of multivariate Student's $t$ random variables, presented in Box, et al. (1994) for example. Let $\mathbf{X}$ be a $d \times 1$ vector of random variables that has the general non-standardized Student's $t$ probability density:

$$
\begin{aligned}
h(\mathbf{x} ; \mu, S, \nu)= & (2 \pi)^{-d / 2}\{\operatorname{det}(S)\}^{-1 / 2}\left(\frac{\nu}{2}\right)^{-d / 2} \Gamma\left(\frac{\nu+d}{2}\right) \Gamma\left(\frac{\nu}{2}\right)^{-1} \\
& \cdot\left(1+\frac{(\mathbf{x}-\mu)^{\prime} S^{-1}(\mathbf{x}-\mu)}{\nu}\right)^{-(\nu+d) / 2}
\end{aligned}
$$

where $\mu$ is the mean vector, $S$ is the scale matrix and $\nu$ is the degrees of freedom. Let us partition $\mathbf{x}$ as $\left[\mathbf{x}_{1}^{\prime}, \mathbf{x}_{2}^{\prime}\right]^{\prime}$, with $\mathbf{x}_{1}$ being a $d_{1} \times 1$ vector and $\mathbf{x}_{2}$ being a $d_{2} \times 1$ vector, and $d_{1}+d_{2}=d$. Similarly partition the location vector, $\mu=\left[\mu_{1}^{\prime}, \mu_{2}^{\prime}\right]^{\prime}$, and the scale matrix:

$$
S=\left[\begin{array}{ll}
S_{11} & S_{12} \\
S_{12}^{\prime} & S_{22}
\end{array}\right]
$$

Then the conditional density of $\mathbf{X}_{2}$ given $\mathbf{X}_{1}=\mathbf{x}_{1}$ is $f\left(\mathbf{x}_{2} \mid \mathbf{x}_{1} ; \Sigma, \nu\right)=$

$$
h\left(\mathbf{x}_{2} ; \mu_{2}+S_{12}^{\prime} S_{11}^{-1}\left(\mathbf{x}_{1}-\mu_{1}\right), \frac{\nu+\left(\mathbf{x}_{1}-\mu_{1}\right)^{\prime} S_{11}^{-1}\left(\mathbf{x}_{1}-\mu_{1}\right)}{\nu+d_{1}}\left(S_{22}-S_{12}^{\prime} S_{11}^{-1} S_{12}\right), \nu+d_{1}\right) .
$$


The Student's $t$ copula has two key inputs to be estimated; the correlation matrix $\Sigma=S \nu /(\nu-2)$ and the degrees of freedom parameter $\nu$. Unfortunately no closed-form expression exists for the MLE of either of these and so numerical methods must be employed. This can cause difficulties when the dimension of the problem is greater than about 5 , as the total number of parameters to be estimated is $d(d-1) / 2+1$, which equals 11 for $d=5$, and rises to 436 for $d=30$. To get around this, we instead use $\hat{\Sigma}$ as our estimate of $\Sigma$, where $\hat{\Sigma}$ is the sample correlation matrix of $\left[T_{\nu_{0}}^{-1}\left(u_{1}\right), \ldots, T_{\nu_{0}}^{-1}\left(u_{d}\right)\right]$, and $\nu_{0}$ is set to 10 . We then estimate $\nu$ using maximum likelihood, imposing $\hat{\Sigma}$ as our estimate of $\Sigma$. We could iterate this procedure, updating our estimates of $\Sigma$ and $\nu$ at each stage. We have experimented with this for realistic simulated data and found changes in the parameter estimates generally only occur beyond the second decimal place, and so we use only one iteration here. The scale matrix is estimated by $\hat{S}=\hat{\Sigma}(\hat{\nu}-2) / \hat{\nu}$

$$
\begin{aligned}
\operatorname{Let} \tilde{Y}_{i t}= & T^{-1}\left(\hat{F}_{i}\left(Y_{i t}\right) ; 0,1, \hat{\nu}\right) \\
\tilde{Y}_{t}= & {\left[\tilde{Y}_{1 t}, \ldots, \tilde{Y}_{d t}\right]^{\prime} } \\
\hat{Z}_{1 t}= & \hat{F}_{1}\left(Y_{1 t}\right) \\
\hat{Z}_{i t}= & T\left(\tilde{Y}_{i t} ; \hat{S}_{[1: i-1,1]}^{\prime} \hat{S}_{[1: i-1,1: i-1]}^{-1} \tilde{Y}_{[1: i-1], t},\right. \\
& \left.\frac{\nu+\tilde{Y}_{[1: i-1], t}^{\prime} \hat{S}_{11}^{-1} \tilde{Y}_{[1: i-1], t}}{\hat{\nu}+i-1}\left(\hat{S}_{22}-\hat{S}_{12}^{\prime} \hat{S}_{11}^{-1} \hat{S}_{12}\right), \hat{\nu}+i-1\right) \\
\text { fori }= & 2,3, \ldots, d .
\end{aligned}
$$

where $T(\cdot ; \mu, s, \nu)$ is the non-standardized Student's $t$ distribution with mean $\mu$, scale matrix $s$ and degrees of freedom parameter $\nu$, and $T^{-1}(\cdot ; \mu, s, \nu)$ is the inverse of $T(\cdot ; \mu, s, \nu)$.

We tested the Student's $t$ copula hypothesis for both raw returns and GARCH filtered returns. In Table 13 we present the results for Test 1 and Test 2 of a bivariate $t$-copula for the same 100 randomly selected pairs of stocks. We find that the Student's $t$ copula is rarely rejected across the 100 pairs of assets considered. This is not surprising given that the bivariate normal copula was also not often rejected for these pairs of assets, as the bivariate $t$-copula nests the bivariate normal copula when $\nu \rightarrow \infty$.

\section{[ INSERT TABLE 13 ABOUT HERE ]}

In Table 14 we present results on the plausibility of the Student's $t$ copula for collections of assets of different dimensions. We considered 5 cases of $d=2,5,10,20,30$. In stark contrast with the results for the normal copula, we find no evidence against the Student's $t$ copula for equity returns, in terms of both raw data and GARCH filtered residuals. For all 5 cases the rejection frequency across the 100 randomly selected collections of assets is less than or equal to the nominal size of the test (0.05), suggesting that the Student's $t$ copula is a reasonable specification. 


\section{[ INSERT TABLE 14 ABOUT HERE ]}

Using univariate GARCH models to remove the conditional variance dynamics increases the average degrees of freedom parameter in all cases, reflecting the fact that the conditional variances of asset returns are positively correlated. The tendency for raw returns to exhibit more joint extreme movements seems to be partially driven by correlated conditional variances, and partially by joint fat tails of the standardized residuals. Overall our results suggest that the Student's $t$ copula is a much better approximation to the true copula of U.S. equity returns than the normal copula, but that the possibility of mis-specification still exists.

Bai (2003) reports a similar conclusion for his study of the univariate distribution of U.S. equity returns. It is important to note, however, that the joint distribution of $d$ equity returns will be multivariate Student's $t$ only if all marginal distributions and the copula have the same degrees of freedom parameter. If any of these are different, then the joint distribution will have Student's $t$ components but will not be multivariate Student's $t$.

\section{Testing Copula Hypotheses for Exchange Rates}

Exchange rate risk is an important source of risk for international investors, and the joint behavior of exchange rates can have a substantial impact on the distribution of returns on an international portfolio. In this section we analyze 20 exchange rates, all against the U.S. dollar, over period 1 January, 1989 to 30 June, 2003, yielding 3717 daily observations. In all cases we analyze the log-difference of the exchange rate. The currencies considered are those from: Austria, Belgium, Finland, France, Germany, Greece, Ireland, Italy, The Netherlands, Portugal, Spain, Australia, Canada, Denmark, Japan, Norway, Singapore, Sweden, Switzerland and the United Kingdom. The first 11 of these countries joined the euro on January 1, 1999, thus the dependence structure of these exchange rates will be different before and after this date. In the following we deal with this by only using data up to December 31, 1997 when 2 or more countries now in the "eurozone" are under analysis. We drop data from the year 1998 as the exchange rates were converging during that year toward their pre-determined euro entry levels, and thus their behavior may not be considered representative of "normal" exchange rate behavior.

\subsection{Testing the normal copula for exchange rates}

We perform similar analyses on these exchange rates to those conducted on equity returns in the previous section. In the simplest case we ignore time variation in the conditional mean and variance of these exchange rates and analyze the plausibility of the normal copula for the unconditional copula. In the second case we employ an $\operatorname{ARMA}(1,1)-\operatorname{GARCH}(1,1)$ filter to account for timevarying conditional means and variances and test the normal copula for the standardized exchange 
rate returns. The final case allows for time-varying conditional means and variances, and a timevarying conditional correlation matrix in the normal copula via the use of the DCC model described in equations (6.1) to (6.3).

We again randomly select 100 collections of exchange rates, of various dimensions, ranging from $d=2$ to $d=20$. In Table 15 we present the results of Test 1 and Test 2 for bivariate normal copula.

\section{[ INSERT TABLE 15 ABOUT HERE ]}

In contrast to the results for equity returns, Table 15 shows that the bivariate normal copula is rejected for many of the pairs of exchange rates considered in this study. Allowing for time variation in the conditional means, variances and correlation reduces the frequency of rejections, but even the most flexible filter considered still yields a rejection frequency between $41 \%$ and $47 \%$.

In Table 16 we examine the impact of increasing the dimension of the group of exchange rates. We find that for group dimension $d=5$ and higher, the rejection frequency is $100 \%$. Thus we have very strong evidence against the normal copula hypothesis for exchange rates.

\section{[ INSERT TABLE 16 ABOUT HERE ]}

\subsection{Testing t-copula for exchange rates}

In Tables 17 and 18 below we examine the goodness of fit of the Student's $t$ copula for these exchange rates. For equity returns there exists published evidence of asymmetries in the dependence structure, but for exchange rates little comparable work has been done, see Patton (2002a) for one study. These tables show that the Student's $t$ copula generally provides a better fit to exchange rate data than the normal copula. For the bivariate case the rejection frequency is less than $40 \%$ for the raw exchange rate returns, and between $14 \%$ and $24 \%$ for the filtered exchange rate returns. As we increase the number of exchange rates under analysis the rejection frequency for raw returns increases $53 \%$ for baskets with $d=15$ exchange rates, and for the full 20-dimensional problem the Student's $t$ copula is rejected. When a GARCH filter is employed the rejection frequency remains around $15 \%$ regardless of the number of exchange rates in the group. For the full 20-dimensional problem using filtered returns the Student's $t$ copula is not rejected.

Overall these results suggest that the Student's $t$ copula provides a reasonable fit to multivariate exchange rate copulas. We would expect that more sophisticated modeling of the copula, allowing for time-varying conditional correlations and degrees of freedom parameter for example, would further reduce the proportion of rejections, though we do not explore this possibility here.

[ INSERT TABLES 17 AND 18 ABOUT HERE ] 


\section{Conclusion}

Recent work on the dependence between financial assets has called into question the assumption of normality for the joint distribution of asset returns. This has prompted research into more flexible models of the dependence structure between financial assets. Unfortunately finance theory does not provide much guidance on the types of dependence structures to expect, and so the assessment of proposed models is essentially an empirical question. In this paper we provide two simple goodness-of-fit tests for models of the dependence structure (copula) of multivariate financial time series. Both tests are easy to compute and are distribution-free. We use these tests to determine whether significant evidences against the normal copula and $t$-copula exist in U.S. equity returns and exchange rates, considering collections of up to 30 assets simultaneously.

The first proposed test is a consistent test that requires the kernel estimation of a multivariate density function and is recommended for low dimension problems only; the second test may not be consistent against all alternatives, but only requires kernel estimation of a univariate density function, and proved very useful when a large number of financial time series is considered. We justify both tests for observable multivariate strictly stationary time series and for standardized innovations of GARCH models. A simulation study demonstrates the efficacy of both tests for realistic sample sizes and data generating processes.

In our study of U.S. equity returns and exchange rates we find substantial evidence against the normal copula for both types of assets, particularly for large collections of assets. Much less evidence was found against the more flexible Student's $t$ copula, suggesting that this model provides a better approximation to the unknown true copula of these assets. Our study suggests that when moving from two to five or ten dimensions one must account for the non-normality of the dependence structure of financial assets. Allowing for univariate non-normality (via a flexible univariate density model, such as the Student's $t$, the $G E D$, a skewed Student's $t$ etc.) and then imposing normality on the copula will not provide an acceptable fit to daily equity return or exchange rate data; a more flexible model of the dependence structure is required. 


\section{Appendix}

Definition 8.1 Let $\left\{\mathbf{Y}_{t}\right\}$ be a strictly stationary stochastic process and $\mathcal{M}_{s}^{t}(\mathbf{Y})$ denote the sigma field generated by $\left(\mathbf{Y}_{s}, \ldots, \mathbf{Y}_{t}\right)$ for $s \leq t$. The process $\left\{\mathbf{Y}_{t}\right\}$ is called absolutely regular (or $\beta-$ mixing) if

$$
\beta_{\tau}=E\left[\sup _{A \in \mathcal{M}_{\tau}^{\infty}(\mathbf{Y})}\left\{\left|P\left(A \mid \mathcal{M}_{-\infty}^{0}(\mathbf{Y})\right)-P(A)\right|\right\}\right] \rightarrow 0 \quad \text { as } \quad \tau \rightarrow \infty
$$

We note that many financial time series models satisfy this absolutely regular condition, including nonlinear ARCH (Masry and Tjøstheim, 1995), GARCH and stochastic volatility (Carrasco and Chen, 2002), and nonlinear diffusion models (Chen et al, 1998). Properties and additional examples of absolutely regular processes can be found in Doukhan (1994).

The first set of conditions is used to establish the asymptotic null distribution of $\bar{I}_{n}$.

(C1) (i) $\left\{Y_{1 t}, \ldots, Y_{d t}\right\}$ is an absolutely regular process with coefficient $\beta_{\tau}=O\left(\rho^{\tau}\right)$ for some $0<$ $\rho<1$; (ii) Let $\mathbf{Z}_{t}=\left(Z_{1 t}, \ldots, Z_{d t}\right)$. Let $f_{1,1+\tau_{1}, 1+\tau_{2}, \ldots, 1+\tau_{l}}^{\mathbf{Z}}(\cdot)$ denote the density function of $\left(\mathbf{Z}_{1}, \mathbf{Z}_{1+\tau_{1}}, \ldots, \mathbf{Z}_{1+\tau_{l}}\right)$ for $1 \leq l \leq 3$. We assume that $f_{\mathbf{Z}}(\cdot)$ is continuous and bounded. In addition, the following Lipschitz type condition holds: $\mid f_{1,1+\tau_{1}, 1+\tau_{2}, \ldots, 1+\tau_{l}}^{\mathbf{Z}}\left(z_{1}+u_{1}, \ldots, z_{l+1}+\right.$ $\left.u_{l+1}\right)-f_{1,1+\tau_{1}, 1+\tau_{2}, \ldots, 1+\tau_{l}}^{\mathbf{Z}}\left(z_{1}, \ldots, z_{l+1}\right) \mid \leq D\left(z_{1}, \ldots, z_{l+1}\right)\|u\|$, where $D(\cdot, \ldots, \cdot)$ is integrable.

(C2) The kernel function $k(\cdot):[-1,1] \rightarrow R^{+}$is a symmetric, bounded, and twice continuously differentiable probability density function such that $\int_{-1}^{1} u^{2} k(u) d u<\infty$;

(C3) $h=O\left(n^{-c}\right)$ for some $0<c<1 / d$.

The second set of conditions is used to show that the asymptotic null distribution of $\widehat{I}_{n}$ is the same as that of $\bar{I}_{n}$.

(A1) $\hat{\alpha} \in \mathcal{A}$ is a parameter estimator such that $\sqrt{n}\left(\hat{\alpha}-\alpha^{*}\right)=O_{p}(1)$, where $\alpha^{*}$ is an interior element of $\mathcal{A}$ and $\alpha^{*}=\alpha_{0}$ under $H_{0}$;

(A2) $C_{0}\left(u_{1}, \ldots, u_{d} ; \alpha\right)$ is twice differentiable.

Proof of Theorem 3.1: The proof consists of two parts. The first part establishes the asymptotic null distribution of $\bar{I}_{n}:\left(n h^{d / 2} \bar{I}_{n}-c_{d n}\right) / \sigma_{d} \rightarrow N(0,1)$ in distribution, where $c_{d n}$ and $\sigma_{d}^{2}$ are defined in Theorem 3.1. In the second part of the proof, we analyze the difference between $\widehat{I}_{n}$ and $\bar{I}_{n}$.

Part I. The proof of this part is the same as that in Fan and Ullah (1999) except that the kernel function in this paper is a boundary kernel. Taking this into account as in Fan and Rilstone (2001) and Hong and Li (2002), one can easily establish the stated result. For space considerations, 
we only provide a detailed derivation of the center term $c_{d n}$. It is well known that the center term is given by the dominant term of $E \bar{I}_{n}=\operatorname{MISE}(\bar{g})$. Ignoring higher order terms, one can show that under $H_{0}$,

$$
\begin{aligned}
E \bar{I}_{n}= & \operatorname{MIVAR}(\bar{g}) \\
= & \frac{1}{n h^{2 d}} \Pi_{j=1}^{d} E\left[\int_{0}^{1} K_{h}^{2}\left(z_{j}, Z_{j t}\right) d z_{i}\right] \\
= & \frac{1}{n h^{2 d}} \Pi_{j=1}^{d} E\left[\int_{0}^{h}\left\{\frac{k\left(\frac{z_{j}-Z_{j t}}{h}\right)}{\int_{-z_{j} / h}^{1} k(u) d u}\right\}^{2} d z_{j}+\int_{h}^{1-h} k^{2}\left(\frac{z_{j}-Z_{j t}}{h}\right) d z_{j}+\int_{1-h}^{1}\left\{\frac{k\left(\frac{z_{j}-Z_{j t}}{h}\right)}{\int_{-1}^{\left(1-z_{j}\right) / h} k(u) d u}\right\}^{2} d z_{j}\right] \\
= & \frac{1}{n h^{2 d}}\left[\int_{0}^{1} \int_{0}^{h}\left\{\frac{k\left(\frac{z-y}{h}\right)}{\int_{-\frac{z}{h}}^{1} k(u) d u}\right\}^{2} d z d y+\int_{0}^{1} \int_{h}^{1-h} k^{2}\left(\frac{z-y}{h}\right) d z d y\right. \\
& \left.+\int_{0}^{1} \int_{1-h}^{1}\left\{\frac{k\left(\frac{z-y}{h}\right)}{\int_{-1}^{\frac{(1-z)}{h}} k(u) d u}\right\}^{d} d z d y\right]^{2} \\
\equiv & \frac{1}{n h^{2 d}}[F+S+T]^{d} .
\end{aligned}
$$

We now establish the dominant terms of $F, S$, and $T$ respectively. First,

$$
\begin{aligned}
F & =\int_{0}^{1} \int_{0}^{h}\left\{\frac{k\left(\frac{z-y}{h}\right)}{\int_{-z / h}^{1} k(u) d u}\right\}^{2} d z d y \\
& =\int_{0}^{h} \frac{1}{\left[\int_{-z / h}^{1} k(u) d u\right]^{2}}\left[\int_{0}^{1} k^{2}\left(\frac{z-y}{h}\right) d y\right] d z \\
& =h \int_{0}^{h} \frac{1}{\left[\int_{-z / h}^{1} k(u) d u\right]^{2}}\left[\int_{(z-1) / h}^{z / h} k^{2}(y) d y\right] d z \\
& =h^{2} \int_{0}^{1} \frac{1}{\left[\int_{-z}^{1} k(u) d u\right]^{2}}\left[\int_{z-h^{-1}}^{z} k^{2}(y) d y\right] d z \\
& =h^{2} \int_{0}^{1} \frac{1}{\left[\int_{-1}^{z} k(u) d u\right]^{2}}\left[\int_{-1}^{z} k^{2}(y) d y\right] d z,
\end{aligned}
$$

we have used the symmetry of $k(\cdot)$ and the fact that for $0 \leq z \leq 1$, it holds that $z-h^{-1} \leq 1-h^{-1} \rightarrow$ $-\infty$ as $n \rightarrow \infty$. Similarly,

$$
\begin{aligned}
S & =\int_{0}^{1} \int_{h}^{1-h} k^{2}\left(\frac{z-y}{h}\right) d z d y \\
& =\int_{h}^{1-h}\left[\int_{0}^{1} k^{2}\left(\frac{z-y}{h}\right) d y\right] d z \\
& =\int_{h}^{1-h}\left[h \int_{(z-1) / h}^{z / h} k^{2}(y) d y\right] d z
\end{aligned}
$$




$$
\begin{aligned}
& =\int_{h}^{1-h}\left[h \int_{-1}^{1} k^{2}(y) d y\right] d z \\
& =h(1-2 h) \int_{-1}^{1} k^{2}(y) d y,
\end{aligned}
$$

where we have used the fact that for $h \leq z \leq(1-h)$, it holds that $1 \leq z / h$ and $(z-1) / h \leq-1$. Finally,

$$
\begin{aligned}
T & =\int_{0}^{1} \int_{1-h}^{1}\left\{\frac{k\left(\frac{z-y}{h}\right)}{\int_{-1}^{(1-z) / h} k(u) d u}\right\}^{2} d z d y \\
& =\int_{1-h}^{1}\left\{\int_{0}^{1} k^{2}\left(\frac{z-y}{h}\right) d y\right\} \frac{1}{\left[\int_{-1}^{(1-z) / h} k(u) d u\right]^{2}} d z \\
& =h \int_{1-h}^{1}\left\{\int_{(z-1) / h}^{z / h} k^{2}(y) d y\right\} \frac{1}{\left[\int_{-1}^{(1-z) / h} k(u) d u\right]^{2}} d z \\
& =h^{2} \int_{0}^{1}\left\{\int_{-z}^{h^{-1}-z} k^{2}(y) d y\right\} \frac{1}{\left[\int_{-1}^{z} k(u) d u\right]^{2}} d z \\
& =h^{2} \int_{0}^{1}\left\{\int_{-1}^{z} k^{2}(y) d y\right\} \frac{1}{\left[\int_{-1}^{z} k(u) d u\right]^{2}} d z,
\end{aligned}
$$

where we have made a change of variables from $z$ to $(1-z) / h$ to obtain the second last equation and used the fact that for $0 \leq z \leq 1$, it holds that $h^{-1}-z \geq h^{-1}-1 \rightarrow \infty$, and the symmetry of $k(\cdot)$.

The center term is obtained from equations (A.1)-(A.4).

Part II. In this part, we will show that $\widehat{I}_{n}-\bar{I}_{n}=o_{p}\left(\left(n h^{d / 2}\right)^{-1}\right)$. The reason for this is that the estimators involved in constructing $\hat{Z}_{1 t}, \ldots, \hat{Z}_{d t}$ are all $n^{-1 / 2}$ consistent. Ignoring higher order terms, we get

$$
\begin{aligned}
\widehat{I}_{n}= & \bar{I}_{n}+\int_{[0,1]^{d}}\left\{\frac{1}{n h^{d}} \sum_{t=1}^{n} \Pi_{j \neq l, j=1}^{d} K_{h}\left(z_{j}, Z_{j t}\right) K_{h, 2}\left(z_{l}, Z_{l t}\right)\left(\hat{Z}_{l t}-Z_{l t}\right)\right\}^{2} d z \\
& +2 \int_{[0,1]^{d}}\left[\frac{1}{n h^{d}} \sum_{t=1}^{n} \Pi_{j=1}^{d} K_{h}\left(z_{j}, Z_{j t}\right)-1\right]\left\{\frac{1}{n h^{d}} \sum_{t=1}^{n} \Pi_{j \neq l, j=1}^{d} K_{h}\left(z_{j}, Z_{j t}\right) K_{h, 2}\left(z_{l}, Z_{l t}\right)\left(\hat{Z}_{l t}-Z_{l t}\right)\right\}^{2} d z
\end{aligned}
$$

where $z=\left(z_{1}, \ldots, z_{d}\right)$ and

$$
K_{h, 2}(x, y)= \begin{cases}-\frac{1}{h} k^{\prime}\left(\frac{x-y}{h}\right) / \int_{-(x / h)}^{1} k(u) d u, & \text { if } x \in[0, h), \\ -\frac{1}{h} k^{\prime}\left(\frac{x-y}{h}\right), & \text { if } x \in[h, 1-h], \\ -\frac{1}{h} k^{\prime}\left(\frac{x-y}{h}\right) / \int_{-1}^{(1-x) / h} k(u) d u, & \text { if } x \in(1-h, 1],\end{cases}
$$

By expanding $\hat{Z}_{l t}-Z_{l t}$ in terms of $\hat{F}_{j}\left(Y_{j t}\right)-F_{j}\left(Y_{j t}\right)$ and $\hat{\alpha}-\alpha_{0}$ and then using the expressions for $\hat{F}_{j}\left(Y_{j t}\right)-F_{j}\left(Y_{j t}\right)$ and the property of $\hat{\alpha}$, one can show by tedious calculations that the last two terms on the right hand side of (A.5) are $o_{p}\left(\left(n h^{d / 2}\right)^{-1}\right)$. 


\section{References}

[1] Ang, A. and J. Chen (2002), "Asymmetric Correlations of Equity Portfolios", Journal of Financial Economics 63, 3, 443-494.

[2] Bai, J. (2003), "Testing Parametric Conditional Distributions of Dynamic Models," forthcoming in Review of Economics and Statistics, 85, 531-549.

[3] Bauwens, L., Laurent, S., and Rombouts, J. V. K., 2003, Multivariate GARCH Models: A Survey, CORE Discussion Paper 2003/31.

[4] Bollerslev, T. and J.M. Wooldridge (1992), "Quasi-maximum likelihood estimation and inference in dynamic models with time-varying covariances," Econometric Reviews, 11, 143-172.

[5] Box, George E. P., Jenkins, Gwilym M. and Reinsel, Gregory C., 1994, Time Series Analysis: Forecasting and Control, Prentice-Hall, New York.

[6] Bouyé, E. , V. Durrleman, A. Nileghbali, G. Riboulet, and T. Roncalli (2000), "Copulas for Finance: A Reading Guide and Some Applications," Manuscript, Financial Econometrics Research Center.

[7] Breymann, W. , A. Dias, and Embrechts, P. (2003), "Dependence Structures for Multivariate High-Frequency Data in Finance," Quantitative Finance 3(1), 1-16.

[8] Carrasco, M. and X. Chen (2002), "Mixing and Moment Properties of Various GARCH and Stochastic Volatility Models," Econometric Theory, 18, 17-39.

[9] Chen, X. and Y. Fan (2002), "Estimation of Copula-Based Semiparametric Time Series Models," Working paper, New York University and Vanderbilt University.

[10] Chen, X., L.P. Hansen and M. Carrasco (1998), "Nonlinearity and Temporal Dependence", working paper, University of Chicago.

[11] Cherubini, U. and E. Luciano (2002), "Multivariate Option Pricing With Copulas," forthcoming in Applied Mathematical Finance.

[12] Cherubini, U. and E. Luciano (2001), "Value at Risk Trade-off and Capital Allocation With Copulas," forthcoming in Economic Notes, 30.

[13] Costinot, A., T. Roncalli, and J. Teiletche (2000): "Revisiting the Dependence Between Financial Markets with Copulas," Working Paper, Crédit Lyonnais.

[14] Dias, A. and P. Embrechts (2003), "Dynamic Copula Models for Multivariate High-Frequency Data in Finance," mimeo.

[15] Diebold, F. X. , T. Gunther, and A. S. Tay (1998), "Evaluating Density Forecasts, with Applications to Financial Risk Management," International Economic Review 39, 863-883.

[16] Doukhan, P. (1994), Mixing: Properties and Examples. New York, Springer.

[17] Duan, J. (2002), "Nonparametric Option Pricing by Transformation," mimeo, University of Toronto.

[18] Embrechts, P. , A. McNeil, and D. Straumann (2002), "Correlation and Dependence Properties in Risk Management: Properties and Pitfalls," in M. Dempster, ed., Risk Management: Value at Risk and Beyond, Cambridge University Press, 176-223.

[19] Embrechts, P. , A. Hoing, and A. Juri (2003), "Using Copulae to Bound the Value-at-Risk for Functions of Dependent Risks," Finance and Stochastics 7(2), 145-167. 
[20] Engle, R. (2002) "Dynamic Conditional Correlation-A Simple Class of Multivariate GARCH Models," Forthcoming in Journal of Business and Economic Statistics.

[21] Engle, R. and K. Sheppard (2001) "Theoretical and Empirical Properties of Dynamic Conditional Correlation Multivariate GARCH," working paper, New York University.

[22] Fan, Y. (1994), "Testing the Goodness of Fit of a Parametric Density Function by Kernel Method," Econometric Theory, 10, 316-356.

[23] Fan, Y. and P. Rilstone (2001), "A Consistent Test for the Parametric Specification of the Hazard Function," Annals of Economics and Finance, 2, 77-96.

[24] Fan, Y. and A. Ullah (1999), "On Goodness-of-fit Tests for Weakly Dependent Processes Using kernel Method," Journal of Nonparametric Statistics, 11, 337-360.

[25] Fermanian, J.-D. (2003), "Goodness of Fit Tests for Copulas", mimeo, CREST.

[26] Fermanian, J.-D. and O. Scaillet (2002), "Nonparametric Estimation of Copulas for Time Series," forthcoming in Journal of Risk.

[27] Frees, Edward W. and E. A. Valdez (1998), "Understanding Relationships Using Copulas," North American Actuarial Journal 2, 1-25.

[28] Frey, R. and A. J. McNeil (2001), "Modeling Dependent Defaults," Working Paper, Department of Mathematics, ETHZ.

[29] Gagliardini, P. and C. Gourieroux (2002), "Duration Time Series Models with Proportional Hazard", working paper, CREST.

[30] Genest, C. , K. Ghoudi, and L.-P. Rivest (1995), "A Semiparametric Estimation Procedure of Dependence Parameters in Multivariate Families of Distributions," Biometrika 82 (3), 543-552.

[31] Hong, Y. and H. Li (2002), "Nonparametric Specification Testing for Continuous-Time Models with Application to Spot Interest Rates," mimeo, Cornell University.

[32] Hu, L. (2002), "Dependence Patterns Across Financial Markets: Methods and Evidence," Manuscript, Yale University and Ohio-State University.

[33] Hull, J. and A. White (1998): "Value at Risk when Daily Changes in Market Variables are not Normally Distributed", Journal of Derivatives, 5, 9-19.

[34] Joe, H. (1997), Multivariate Models and Dependence Concepts, Chapman \& Hall/CRC.

[35] Junker, M. and A. May (2002), "Measurement of Aggregate Risk With Copulas," Preprint, Caesar.

[36] Khmaladze, E. V. (1981), "Martingale approach in the theory of goodness-of-fit tests. Theory of Probability and its Applications 26, 240-257.

[37] Klugman, S. A. and R. Parsa (1999), "Fitting Bivariate Loss Distributions With Copulas," Insurance: Mathematics and Economics 24, 139-148.

[38] Li, David X. (2000), "On Default Correlation: A Copula Function Approach," Journal of Fixed Income $9,43-54$.

[39] Longin, F. and B. Solnik (2001), "Extreme Correlation of International Equity Market," Journal of Finance 56, 649-679. 
[40] Malevergne, Y. and D. Sornette (2003), "Testing the Gaussian Copula Hypothesis for Financial Assets Dependences," Quantitative Finance, 3, 231-250.

[41] Mashal, R. and A. Zeevi (2002), "Beyond Correlation: Extreme Co-movements Between Financial Assets," manuscript, Columbia University.

[42] Masry, E. and D. Tjøstheim (1995), "Nonparametric Estimation and Identification of Nonlinear ARCH Time Series: Strong Convergence and Asymptotic Normality," Econometric Theory, $11,258-289$.

[43] Mills, Frederick C., 1927, The Behavior of Prices, National Bureau of Economic Research, New York.

[44] Müller, Hans-Georg (1991), "Smooth Optimal Kernel Estimators Near Endpoints," Biometrika $78,521-530$.

[45] Nelsen, R. B. (1999), An Introduction to Copulas, Springer.

[46] Patton, A.J. (2002a), "Modeling Time-Varying Exchange Rate Dependence Using the Conditional Copula," Working Paper 01-09, Department of Economics, University of California, San Diego.

[47] Patton, A.J. (2002b), "Estimation of Copula Models for Time Series of Possibly Different Lengths," Working Paper 01-17, Department of Economics, University of California, San Diego.

[48] Patton, A.J. (2004), "On the Importance of Skewness and Asymmetric Dependence in Stock Returns for Asset Allocation," forthcoming, Journal of Financial Econometrics.

[49] Robinson, P. and P. Zaffaroni (2002), "Pseudo-Maximum Likelihood Estimation of ARCH( $\infty)$ Models," working paper, LSE.

[50] Rockinger, M. and E. Jondeau (2002), "Conditional Dependency of Financial Series: An Application of Copulas," Manuscript, HEC School of Management, France.

[51] Rosenberg, J. (2003), "Nonparametric Pricing of Multivariate Contingent Claims," Journal of Derivatives, 10(3), 9-26.

[52] Rosenblatt, M. (1952), "Remarks on a Multivariate Transformation," Annals of Mathematical Statistics 23, 470-472.

[53] Schönbucher, P. and D. Schubert (2001), "Copula Dependent Default Risk in Intensity Models", mimeo, University of Bonn.

[54] Sklar, A. (1959), "Fonctions de r'epartition 'a $n$ dimensions et leurs marges," Publ. Inst. Statis. Univ. Paris 8, 229-231.

[55] Stute, W. , S. Thies, and L. Zhu (1998), "Model Checks for Regression: AN Innovation Process Approach," Annals of Statistics, 26, 1916-1934.

[56] Thompson, S. (2002), "Evaluating the Goodness of Fit of Conditional Distributions, with an Application to Affine Term Structure Models," forthcoming in Review of Financial Studies.

[57] Tse, Y. K., 2000, A Test for Constant Correlations in a Multivariate GARCH Model, Journal of Econometrics, 98, 107-127. 


\begin{tabular}{lccll}
\hline \multicolumn{5}{c}{ Table 1: Tail dependence in the bivariate $t$ copula } \\
\hline \multicolumn{5}{c}{$\rho$} \\
\hline$\nu$ & 0 & 0.2 & 0.5 & 0.8 \\
\hline 4 & 0.0378 & 0.0637 & 0.1266 & 0.2448 \\
6 & 0.0166 & 0.0338 & 0.0852 & 0.2035 \\
10 & 0.0034 & 0.0102 & 0.0409 & 0.1463 \\
$\infty$ & 0 & 0 & 0 & 0 \\
\hline
\end{tabular}

Note: This table presents the tail dependence implied by the Student's $t$ copula for various levels of correlation and various degrees of freedom values.

\begin{tabular}{ccccccc}
\hline \multicolumn{2}{c}{ Table 2: KLIC between Normal and Student's $t$ copula, $\rho=0.5$} \\
\cline { 2 - 7 } Dimension & 4 & 6 & 10 & 20 & 30 & 50 \\
\hline $1^{*}$ & 0.3853 & 0.2171 & 0.1161 & 0.0535 & 0.0347 & 0.0204 \\
2 & 7.5864 & 6.8612 & 6.3660 & 5.9574 & 5.8969 & 5.7773 \\
3 & 7.8933 & 7.0550 & 6.4386 & 5.9855 & 5.9196 & 5.8452 \\
5 & 8.5726 & 7.4096 & 6.6752 & 6.1324 & 5.9730 & 5.9043 \\
10 & 10.2132 & 8.4966 & 7.2460 & 6.4293 & 6.1142 & 5.9848 \\
20 & 13.4706 & 10.6224 & 8.4791 & 6.9961 & 6.5758 & 6.1844 \\
30 & 16.6329 & 12.5668 & 9.6355 & 7.6430 & 6.9304 & 6.4101 \\
\hline
\end{tabular}

Note: This table presents the Kullback-Leibler Information Criterion (KLIC) between the Normal and Student's $t$ copulas. The KLIC between these two copulas is computed as:

$$
K L I C\left(c_{\text {normal }}(\rho): c_{t}(\rho, \nu)\right)=\int \log \left(\frac{c_{\text {normal }}(\mathbf{u} ; \Sigma)}{c_{t}(\mathbf{u} ; \Sigma, \nu)}\right) c_{\text {normal }}(\mathbf{u} ; \Sigma) d \mathbf{u}
$$

where $\Sigma$ is a matrix with ones on diagonal and $\rho$ for all off-diagonal elements. The integration is over $[0,1]^{d}$, where $d$ is the dimension of the copulas being compared. We obtained the above estimates of the KLIC by drawing $n=10,000$ observations from the $d$-dimensional Normal copula with correlation matrix $\Sigma$, denoted $\left\{\mathbf{u}_{t}\right\}_{t=1}^{n}$, and then computing:

$$
\widehat{K L I C}\left(c_{\text {normal }}(\rho): c_{t}(\rho, \nu)\right)=n^{-1} \sum_{t=1}^{n} \log \left(\frac{c_{\text {normal }}\left(\mathbf{u}_{t} ; \Sigma\right)}{c_{t}\left(\mathbf{u}_{t} ; \Sigma, \nu\right)}\right)
$$

*For the purposes of comparison we present the KLIC between the univariate normal and Student's $t$ probability densities. 


\begin{tabular}{lccccc}
\hline \multicolumn{6}{c}{ Table 3: Probability of rejecting the null using Test 1 } \\
\hline $\mathrm{p}$ & 0 & 0.25 & 0.5 & 0.75 & 1 \\
\hline $\mathrm{N}$ & \multicolumn{6}{c}{ Correlation $=0.1$} \\
\hline 500 & 0.000 & 0.000 & 0.016 & 0.000 & 0.000 \\
2500 & 0.024 & 0.032 & 0.000 & 0.096 & 0.536 \\
5000 & 0.056 & 0.000 & 0.064 & 0.736 & 1.000 \\
\hline \multicolumn{6}{c}{ Correlation $=0.5$} \\
\hline 500 & 0.000 & 0.000 & 0.000 & 0.0000 & 0.000 \\
2500 & 0.032 & 0.000 & 0.000 & 0.096 & 0.520 \\
5000 & 0.032 & 0.000 & 0.040 & 0.704 & 0.992 \\
\hline \multicolumn{6}{c}{ Correlation $=0.9$} \\
\hline 500 & 0.000 & 0.000 & 0.000 & 0.000 & 0.024 \\
2500 & 0.000 & 0.008 & 0.016 & 0.256 & 0.776 \\
5000 & 0.024 & 0.008 & 0.120 & 0.792 & 1.000 \\
\hline
\end{tabular}

Note: This table presents the results of a Monte Carlo study of the size and power of Test 1 . The null hypothesis is that the copula is normal. The DGP is a mixture of a normal and a $t_{4}$ copula: $C=$ $(1-p) C_{\text {normal }}+p C_{t_{4}}$, so the case that $p=0$ corresponds to the case that the DGP satisfies the null hypothesis. The marginal distributions were set to be iid $N(0,1)$. The nominal size in all cases is 0.05 . The number of replications was 500. The dimension considered was 2.

\begin{tabular}{lccccc}
\hline \multicolumn{6}{c}{ Table 4: Probability of rejecting the null using Test 2 } \\
\hline $\mathrm{p}$ & 0 & 0.25 & 0.5 & 0.75 & 1 \\
\hline $\mathrm{N}$ & \multicolumn{6}{c}{ Correlation $=0.1$} \\
\hline 500 & 0.008 & 0.000 & 0.000 & 0.008 & 0.000 \\
2500 & 0.016 & 0.000 & 0.008 & 0.120 & 0.512 \\
5000 & 0.024 & 0.008 & 0.048 & 0.752 & 0.992 \\
\hline \multicolumn{6}{c}{ Correlation $=0.5$} \\
\hline 500 & 0.008 & 0.000 & 0.000 & 0.000 & 0.000 \\
2500 & 0.024 & 0.008 & 0.000 & 0.144 & 0.680 \\
5000 & 0.040 & 0.008 & 0.152 & 0.800 & 1.000 \\
\hline \multicolumn{6}{c}{ Correlation $=0.9$} \\
\hline 500 & 0.000 & 0.000 & 0.000 & 0.000 & 0.048 \\
2500 & 0.016 & 0.008 & 0.008 & 0.336 & 0.816 \\
5000 & 0.024 & 0.024 & 0.192 & 0.872 & 1.000 \\
\hline
\end{tabular}

Note: This table presents the results of a Monte Carlo study of the size and power of Test 2. The null hypothesis is that the copula is normal. The DGP is a mixture of a normal and a $t_{4}$ copula: $C=$ $(1-p) C_{\text {normal }}+p C_{t_{4}}$, so the case that $p=0$ corresponds to the case that the DGP satisfies the null hypothesis. The marginal distributions were set to be iid $N(0,1)$. The nominal size in all cases is 0.05 . The number of replications was 500 . The dimension considered was 2. 


\begin{tabular}{lccccc}
\hline \multicolumn{6}{c}{ Table 5: Probability of rejecting the null using Test 1 } \\
\hline $\mathrm{p}$ & 0 & 0.25 & 0.5 & 0.75 & 1 \\
\hline $\mathrm{N}$ & \multicolumn{6}{c}{ Correlation $=0.1$} \\
\hline 500 & 0.000 & 0.000 & 0.000 & 0.000 & 0.000 \\
2500 & 0.004 & 0.004 & 0.008 & 0.064 & 0.568 \\
5000 & 0.052 & 0.000 & 0.048 & 0.704 & 1.000 \\
\hline \multicolumn{6}{c}{ Correlation $=0.5$} \\
\hline 500 & 0.000 & 0.000 & 0.000 & 0.000 & 0.000 \\
2500 & 0.024 & 0.016 & 0.000 & 0.084 & 0.480 \\
5000 & 0.040 & 0.008 & 0.068 & 0.684 & 0.992 \\
\hline \multicolumn{6}{c}{ Correlation $=0.9$} \\
\hline 500 & 0.000 & 0.000 & 0.000 & 0.000 & 0.020 \\
2500 & 0.000 & 0.004 & 0.016 & 0.228 & 0.728 \\
5000 & 0.008 & 0.008 & 0.188 & 0.828 & 1.000 \\
\hline
\end{tabular}

Note: This table presents the results of a Monte Carlo study of the size and power of Test 1 . The null hypothesis is that the copula is normal. The DGP is a mixture of a normal and a $t_{4}$ copula: $C=(1-$ p) $C_{\text {normal }}+p C_{t_{4}}$, so the case that $p=0$ corresponds to the case that the DGP satisfies the null hypothesis. The marginal distributions are assumed to be $\operatorname{AR}(1)-\operatorname{GARCH}(1,1)$ processes with Normal innovations. The nominal size in all cases is 0.05 . The number of replications was 500 . The dimension considered was 2 .

\begin{tabular}{lccccc}
\hline \multicolumn{6}{c}{ Table 6: Probability of rejecting the null using Test 2 } \\
\hline $\mathrm{p}$ & 0 & 0.25 & 0.5 & 0.75 & 1 \\
\hline $\mathrm{N}$ & \multicolumn{6}{c}{ Correlation $=0.1$} \\
\hline 500 & 0.004 & 0.012 & 0.004 & 0.004 & 0.004 \\
2500 & 0.020 & 0.004 & 0.016 & 0.140 & 0.608 \\
5000 & 0.032 & 0.020 & 0.092 & 0.716 & 0.996 \\
\hline \multicolumn{6}{c}{ Correlation $=0.5$} \\
\hline 500 & 0.016 & 0.004 & 0.000 & 0.000 & 0.008 \\
2500 & 0.024 & 0.000 & 0.012 & 0.208 & 0.668 \\
5000 & 0.024 & 0.020 & 0.092 & 0.772 & 1.000 \\
\hline \multicolumn{6}{c}{ Correlation $=0.9$} \\
\hline 500 & 0.000 & 0.008 & 0.008 & 0.008 & 0.080 \\
2500 & 0.020 & 0.008 & 0.072 & 0.380 & 0.776 \\
5000 & 0.032 & 0.012 & 0.288 & 0.836 & 1.000 \\
\hline
\end{tabular}

Note: This table presents the results of a Monte Carlo study of the size and power of Test 2. The null hypothesis is that the copula is normal. The DGP is a mixture of a normal and a $t_{4}$ copula: $C=(1-$ p) $C_{\text {normal }}+p C_{t_{4}}$, so the case that $p=0$ corresponds to the case that the DGP satisfies the null hypothesis. The marginal distributions are assumed to be $\operatorname{AR}(1)-\operatorname{GARCH}(1,1)$ processes with Normal innovations. The nominal size in all cases is 0.05 . The number of replications was 500 . The dimension of the system considered is 2 . 


\begin{tabular}{lccccc}
\hline \multicolumn{6}{c}{ Table 7: Probability of rejecting the null using Test 2 } \\
\hline $\mathrm{p}$ & 0 & 0.25 & 0.5 & 0.75 & 1 \\
\hline $\mathrm{N}$ & \multicolumn{6}{c}{ Correlation $=0.1$} \\
\hline 500 & 0.00 & 0.06 & 0.45 & 1.00 & 1.00 \\
2500 & 0.04 & 0.74 & 1.00 & 1.00 & 1.00 \\
5000 & 0.02 & 1.00 & 1.00 & 1.00 & 1.00 \\
\hline \multicolumn{6}{c}{ Correlation $=0.5$} \\
\hline 500 & 0.00 & 0.08 & 0.58 & 0.98 & 1.00 \\
2500 & 0.00 & 0.69 & 1.00 & 1.00 & 1.00 \\
5000 & 0.08 & 0.99 & 1.00 & 1.00 & 1.00 \\
\hline \multicolumn{6}{c}{ Correlation $=0.9$} \\
\hline 500 & 0.04 & 0.18 & 0.68 & 0.99 & 1.00 \\
2500 & 0.04 & 0.79 & 1.00 & 1.00 & 1.00 \\
5000 & 0.00 & 1.00 & 1.00 & 1.00 & 1.00 \\
\hline
\end{tabular}

Note: This table presents the results of a Monte Carlo study of the size and power of Test 2. The null hypothesis is that the copula is normal. The DGP is a mixture of a normal and a $t_{4}$ copula: $C=$ $(1-p) C_{\text {normal }}+p C_{t_{4}}$, so the case that $p=0$ corresponds to the case that the DGP satisfies the null hypothesis. The marginal distributions were set to be iid $N(0,1)$. The nominal size in all cases is 0.05 . The number of replications was 100 . The dimension of the system considered is 5 .

\begin{tabular}{lccccc}
\hline \multicolumn{6}{c}{ Table 8: Probability of rejecting the null using Test 2 } \\
\hline $\mathrm{p}$ & 0 & 0.25 & 0.5 & 0.75 & 1 \\
\hline $\mathrm{N}$ & \multicolumn{6}{c}{ Correlation $=0.1$} \\
\hline 500 & 0.00 & 0.04 & 0.47 & 0.98 & 1.00 \\
2500 & 0.06 & 0.81 & 1.00 & 1.00 & 1.00 \\
5000 & 0.02 & 0.98 & 1.00 & 1.00 & 1.00 \\
\hline \multicolumn{6}{c}{ Correlation $=0.5$} \\
\hline 500 & 0.02 & 0.05 & 0.48 & 0.94 & 1.00 \\
2500 & 0.00 & 0.76 & 1.00 & 1.00 & 1.00 \\
5000 & 0.04 & 1.00 & 1.00 & 1.00 & 1.00 \\
\hline \multicolumn{6}{c}{ Correlation $=0.9$} \\
\hline 500 & 0.02 & 0.30 & 0.78 & 0.98 & 1.00 \\
2500 & 0.02 & 0.84 & 1.00 & 1.00 & 1.00 \\
5000 & 0.06 & 0.99 & 1.00 & 1.00 & 1.00 \\
\hline
\end{tabular}

Note: This table presents the results of a Monte Carlo study of the size and power of Test 2. The null hypothesis is that the copula is normal. The DGP is a mixture of a normal and a $t_{4}$ copula: $C=(1-$ p) $C_{\text {normal }}+p C_{t_{4}}$, so the case that $p=0$ corresponds to the case that the DGP satisfies the null hypothesis. The marginal distributions are assumed to be $\operatorname{AR}(1)-\operatorname{GARCH}(1,1)$ processes with Normal innovations. The nominal size in all cases is 0.05 . The number of replications was 100 . The dimension of the system considered is 5 . 


\begin{tabular}{lccccc}
\hline \multicolumn{6}{l}{ Table 9: Probability of rejecting the null using Test 2 } \\
\hline $\mathrm{p}$ & 0 & 0.25 & 0.5 & 0.75 & 1 \\
\hline $\mathrm{N}$ & \multicolumn{6}{c}{ Correlation $=0.1$} \\
\hline 500 & 0.16 & 1.00 & 1.00 & 1.00 & 1.00 \\
2500 & 0.07 & 1.00 & 1.00 & 1.00 & 1.00 \\
5000 & 0.05 & 1.00 & 1.00 & 1.00 & 1.00 \\
\hline \multicolumn{6}{c}{ Correlation $=0.5$} \\
\hline 500 & 0.12 & 1.00 & 1.00 & 1.00 & 1.00 \\
2500 & 0.03 & 1.00 & 1.00 & 1.00 & 1.00 \\
5000 & 0.03 & 1.00 & 1.00 & 1.00 & 1.00 \\
\hline \multicolumn{6}{c}{ Correlation $=0.9$} \\
\hline 500 & 0.15 & 1.00 & 1.00 & 1.00 & 1.00 \\
2500 & 0.06 & 1.00 & 1.00 & 1.00 & 1.00 \\
5000 & 0.08 & 1.00 & 1.00 & 1.00 & 1.00 \\
\hline
\end{tabular}

Note: This table presents the results of a Monte Carlo study of the size and power of Test 2. The null hypothesis is that the copula is normal. The DGP is a mixture of a normal and a $t_{4}$ copula: $C=$ $(1-p) C_{\text {normal }}+p C_{t_{4}}$, so the case that $p=0$ corresponds to the case that the DGP satisfies the null hypothesis. The marginal distributions were set to be iid $N(0,1)$. The nominal size in all cases is 0.05 . The number of replications was 100 . The dimension of the system considered is 30 .

\begin{tabular}{lccccc}
\hline \multicolumn{7}{c}{ Table 10: Probability of rejecting the null using Test 2 } \\
\hline $\mathrm{p}$ & 0 & 0.25 & 0.5 & 0.75 & 1 \\
\hline $\mathrm{N}$ & \multicolumn{6}{c}{ Correlation $=0.1$} \\
\hline 500 & 0.09 & 1.00 & 1.00 & 1.00 & 1.00 \\
2500 & 0.00 & 1.00 & 1.00 & 1.00 & 1.00 \\
5000 & 0.00 & 1.00 & 1.00 & 1.00 & 1.00 \\
\hline \multicolumn{6}{c}{ Correlation $=0.5$} \\
\hline 500 & 0.05 & 1.00 & 1.00 & 1.00 & 1.00 \\
2500 & 0.02 & 1.00 & 1.00 & 1.00 & 1.00 \\
5000 & 0.04 & 1.00 & 1.00 & 1.00 & 1.00 \\
\hline \multicolumn{6}{c}{ Correlation $=0.9$} \\
\hline 500 & 0.24 & 1.00 & 1.00 & 1.00 & 1.00 \\
2500 & 0.07 & 1.00 & 1.00 & 1.00 & 1.00 \\
5000 & 0.06 & 1.00 & 1.00 & 1.00 & 1.00 \\
\hline \multicolumn{7}{c}{}
\end{tabular}

Note: This table presents the results of a Monte Carlo study of the size and power of Test 2. The null hypothesis is that the copula is normal. The DGP is a mixture of a normal and a $t_{4}$ copula: $C=(1-$ p) $C_{\text {normal }}+p C_{t_{4}}$, so the case that $p=0$ corresponds to the case that the DGP satisfies the null hypothesis. The marginal distributions are assumed to be $\operatorname{AR}(1)-\operatorname{GARCH}(1,1)$ processes with Normal innovations. The nominal size in all cases is 0.05 . The number of replications was 100 . The dimension of the system considered is 30 . 


\begin{tabular}{cccccc}
\hline \multicolumn{5}{c}{ Table 11: Proportion of times the bivariate Normal Copula can be rejected } \\
\hline \multicolumn{2}{c}{ Raw returns } & \multicolumn{2}{c}{ GARCH filter } & \multicolumn{2}{c}{ GARCH+DCC filter } \\
\hline Test 1 & Test 2 & Test 1 & Test 2 & Test 1 & Test 2 \\
0.01 & 0.01 & 0.00 & 0.03 & 0.01 & 0.03 \\
\hline
\end{tabular}

Note: This table presents the proportion of times the bivariate normal copula was rejected for a randomly selected collection of 100 pairs of equities.

\begin{tabular}{cccc}
\hline \multicolumn{4}{c}{ Table 12: Proportion of times the Normal Copula can be rejected } \\
\hline Number of assets & Raw returns & GARCH filter & GARCH+DCC filter \\
\hline 2 & 0.03 & 0.01 & 0.02 \\
3 & 0.59 & 0.17 & 0.12 \\
5 & 1.00 & 0.81 & 0.80 \\
10 & 1.00 & 1.00 & 1.00 \\
15 & 1.00 & 1.00 & 1.00 \\
20 & 1.00 & 1.00 & 1.00 \\
25 & 1.00 & 1.00 & 1.00 \\
30 & 1.00 & 1.00 & 1.00 \\
\hline
\end{tabular}

Note: This table presents the proportion of times the normal copula was rejected for a randomly selected collection of 100 groups of equities, with the size of the group ranging from 2 to 30. For the group of size 30 there was only one to consider.

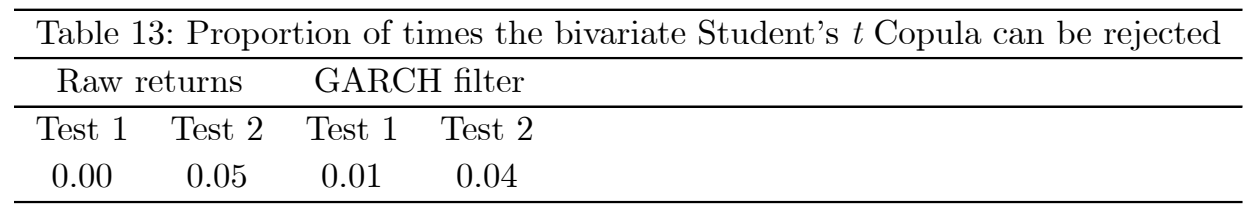

Note: This table presents the proportion of times the bivariate Student's $t$ copula was rejected for a randomly selected collection of 100 pairs of equities.

\begin{tabular}{ccc}
\hline \multicolumn{3}{c}{ Table 14: Proportion of times the Student's $t$ Copula can be rejected } \\
\hline Number of assets & Raw returns & GARCH filter \\
\hline 2 & 0.05 & 0.04 \\
5 & 0.00 & 0.01 \\
10 & 0.01 & 0.01 \\
20 & 0.02 & 0.00 \\
30 & 0.00 & 0.00 \\
\hline
\end{tabular}

Note: This table presents the proportion of times the Student's $t$ copula was rejected for a randomly selected collection of 100 groups of equities, with the size of the group ranging from 2 to 30 . For the group of size 30 there was only one to consider. 


\begin{tabular}{cccccc}
\hline \multicolumn{3}{c}{ Table 15: Proportion of times the bivariate Normal Copula can be rejected } \\
\hline \multicolumn{2}{c}{ Raw returns } & \multicolumn{2}{c}{ GARCH filter } & \multicolumn{2}{c}{ GARCH+DCC filter } \\
\hline Test 1 & Test 2 & Test 1 & Test 2 & Test 1 & Test 2 \\
0.75 & 0.74 & 0.64 & 0.66 & 0.41 & 0.47 \\
\hline
\end{tabular}

Note: This table presents the proportion of times the bivariate normal copula was rejected for a randomly selected collection of 100 pairs of exchange rates.

\begin{tabular}{cccc}
\hline \multicolumn{4}{c}{ Table 16: Proportion of times the Normal Copula can be rejected } \\
\hline Number of assets & Raw returns & GARCH filter & GARCH+DCC filter \\
\hline 2 & 0.74 & 0.66 & 0.47 \\
5 & 1.00 & 1.00 & 1.00 \\
10 & 1.00 & 1.00 & 1.00 \\
15 & 1.00 & 1.00 & 1.00 \\
20 & 1.00 & 1.00 & 1.00 \\
\hline
\end{tabular}

Note: This table presents the proportion of times the normal copula was rejected for a randomly selected collection of 100 groups of exchange rates, with the size of the group ranging from 2 to 20 . For the group of size 20 there was only one to consider.

\begin{tabular}{cc|cc}
\hline \multicolumn{4}{c}{ Table 17: Proportion of times the bivariate Student's $t$ Copula can be rejected } \\
\hline \multicolumn{2}{c|}{ Raw returns } & \multicolumn{3}{c}{ GARCH filter } \\
\hline Test 1 & Test 2 & Test 1 & Test 2 \\
0.38 & 0.37 & 0.24 & 0.14 \\
\hline
\end{tabular}

Note: This table presents the proportion of times the bivariate Student's $t$ copula was rejected for a randomly selected collection of 100 pairs of exchange rates.

\begin{tabular}{ccc}
\hline \multicolumn{3}{c}{ Table 18: Proportion of times the Student's $t$ Copula can be rejected } \\
\hline Number of assets & Raw returns & GARCH filter \\
\hline 2 & 0.37 & 0.14 \\
5 & 0.26 & 0.13 \\
10 & 0.32 & 0.18 \\
15 & 0.53 & 0.17 \\
20 & 1.00 & 0.00 \\
\hline
\end{tabular}

Note: This table presents the proportion of times the Student's $t$ copula was rejected for a randomly selected collection of 100 groups of exchange rates, with the size of the group ranging from 2 to 20 . For the group of size 20 there was only one to consider. 